\begin{abstract}
BLURRED FINGERPRINT IMAGE ENHANCEMENT: ALGORITHM ANALYSIS AND PERFORMANCE EVALUATION
\end{abstract}

by Annatoma Arif

The Automatic Fingerprint Matching (AFM) uses similarity score between an input and a reference fingerprint images to match fingerprints and the similarity score can be determined with a Minutiae Extraction Algorithm (MEA), which extracts minutiae of input and reference fingerprints. The performance of MEA depends on the quality of input fingerprint images. In case of blurred input fingerprint images, it becomes difficult to obtain a legitimate similarity score used in the AFM process. Therefore, an image enhancement algorithm must be incorporated with MEA to improve the performance of AFM process. In this study, good quality input fingerprint images have been intentionally blurred. Different enhancement algorithms are used to enhance the quality of blurred input fingerprint images. The performance of enhancement algorithms is analyzed and evaluated using the similarity score of extracted minutiae. Experimental results show that Volterra filter significantly enhances the quality of blurred input fingerprint images than other linear filters such as Laplacian and Gabor filters considered in this study. 
This thesis titled

\title{
BLURRED FINGERPRINT IMAGE ENHANCEMENT: ALGORITHM ANALYSIS AND
} PERFORMANCE EVALUATION

\author{
by \\ Annatoma Arif \\ has been approved for publication by \\ College of Engineering and Computing \\ and \\ Department of \\ Electrical and Computer Engineering
}

Advisor: Dr. Chi Hao Cheng

$\overline{\text { Reader: Dr. Qihou Zhou }}$

$\overline{\text { Reader: Dr. Yamuna Rajasekhar }}$

C2016 Annatoma Arif 


\section{Table of Contents}

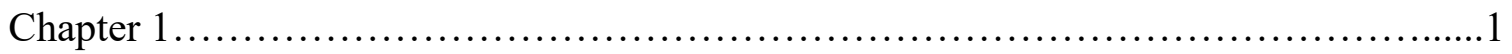

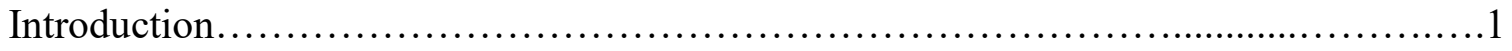

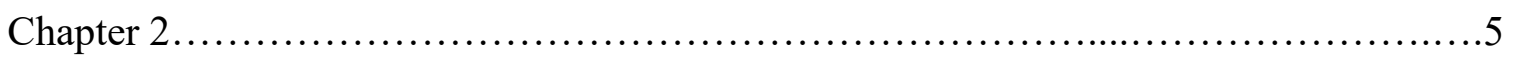

Theoretical Background......................................................

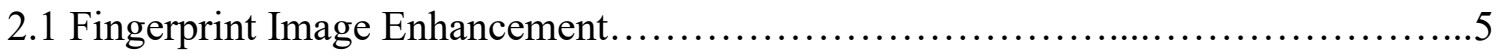

2.1.1 Gabor Filter................................................................

2.1.2 Laplacian Filter...................................................9

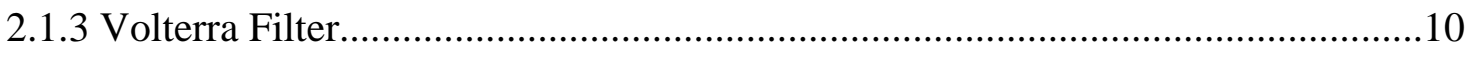

2.2 Evaluation Method.............................................................. 11

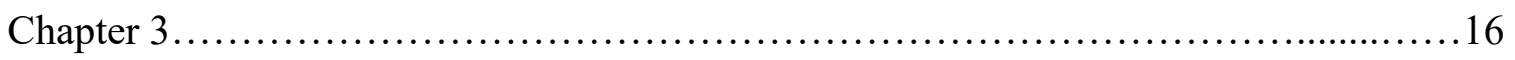

Experimental Results...................................................... 16

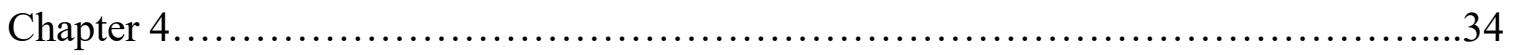

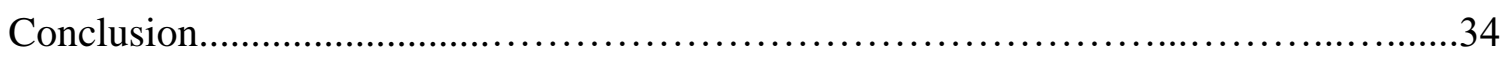

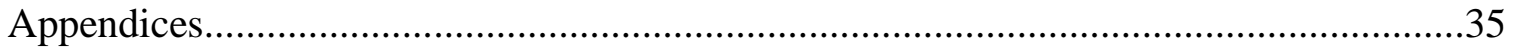

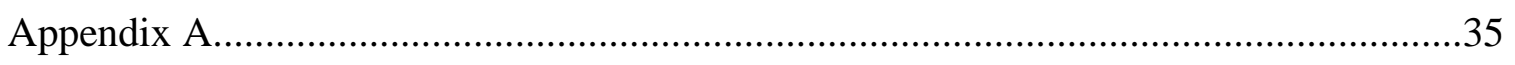

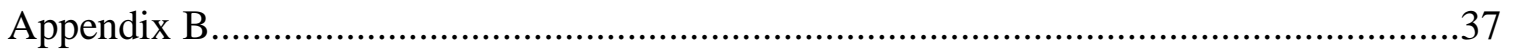

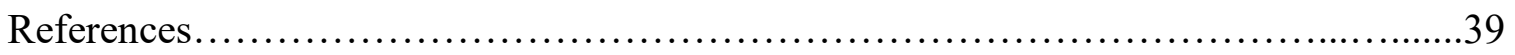




\section{List of Tables}

Table 3.1: Mean Values of Similarity Score between Reference and Enhanced (Processing the Blurred (using a Gaussian Filter (size: $3 \times 3$ ) Fingerprint Images with Different Combination of Volterra Filter Coefficients) Fingerprint Images.......................................16 Table 3.2: Mean Values of Similarity Score between Reference and Unenhanced (Blurred by Gaussian Filter (size: $3 \times 3,5 \times 5,7 \times 7$ ), Average Filter (size: $3 \times 3,5 \times 5,7 \times 7$ ) and Corrupted by noise with SNR 5, 10 and $20 \mathrm{~dB}$ ) Matched Fingerprint Images and Mean Values of Similarity Score between Reference and Enhanced (using Different Enhancement Algorithms on Blurred/Corrupted Images) Matched Fingerprint

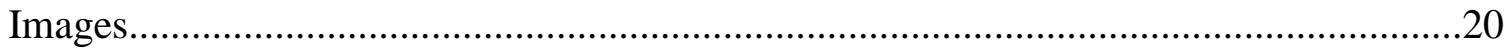

Table 3.3: Mean Values of Similarity Score between Reference and Unenhanced (Blurred by Gaussian Filter (size: $3 \times 3,5 \times 5,7 \times 7$ ), Average Filter (size: $3 \times 3,5 \times 5,7 \times 7$ ) and Corrupted by noise with SNR 5,10 and $20 \mathrm{~dB}$ ) Miss-matched Fingerprint Images and Mean Values of Similarity Score between Reference and Enhanced (using Different Enhancement Algorithms on Blurred/Corrupted Images) Miss-matched Fingerprint

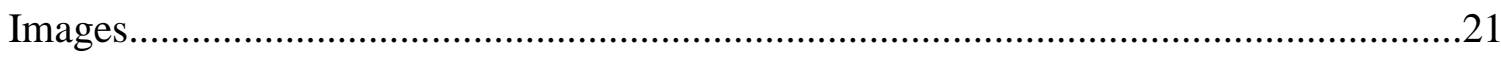

Table 3.4: Comparison of Probability of Error (FNMR and FMR) .................................32

Table B-1: Values of QF for Different Fingerprint Image Enhancement Algorithms........37 


\section{List of Figures}

Figure 1.1: Fingerprint classification: (a) Arch, (b) Tented arch, (c) Right loop, (d) Left loop, (e) Whorl, (f) Twin loop [7] ..............................................................................

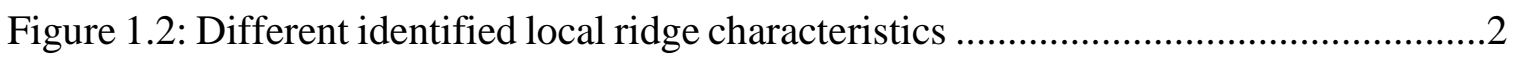

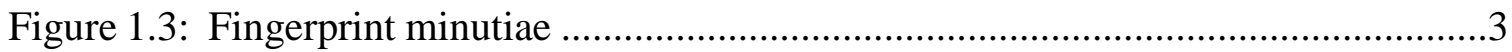

Figure 1.4: (a) Good quality fingerprint images and (b) blurred fingerprint Images.............4

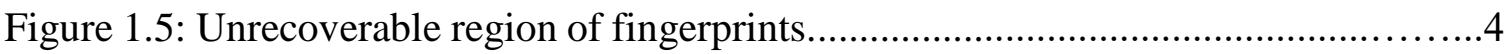

Figure 2.1: Steps of using Gabor filter as fingerprint enhancement algorithm......................5

Figure 2.2: Original image and normalized image $\left(\mathrm{M}_{0}=100\right.$ and $\left.\mathrm{VAR}_{0}=100\right) \ldots \ldots \ldots \ldots \ldots \ldots . . .6$

Figure 2.3: Orientation field estimation of input fingerprint image ......................................

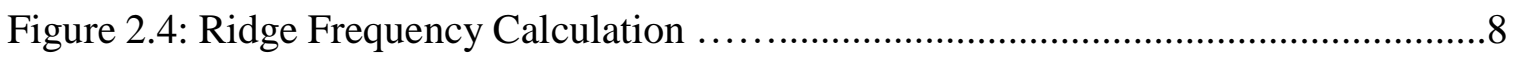

Figure 2.5: Block diagram of Volterra filter as a fingerprint enhancement algorithm..........11

Figure 2.6: A block diagram representing the evaluation method of different enhancement

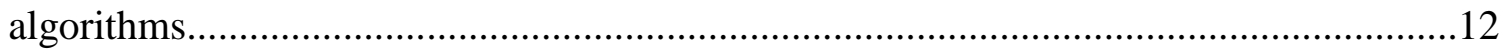

Figure 2.7: Pixel values of a $3 \times 3$ window for (a) ridge ending and (b) bifurcation of a

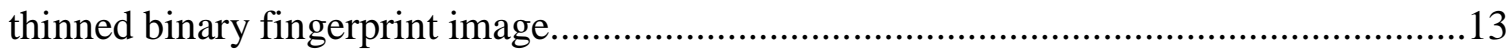

Figure 2.8: Minutiae extraction: (a) Input gray scale image, (b) Binary image, (c) Thinned binary image and (d) Extracted minutiae.........................................................................14

Figure 3.1: Output fingerprint images after using different enhancement algorithms on blurred (using Gaussian filter (size: $3 \times 3,5 \times 5,7 \times 7)$ ) input fingerprint images..........18 Figure 3.2: Output fingerprint images after using different enhancement algorithms on blurred (using Average filter (size: $3 \times 3,5 \times 5,7 \times 7$ )) input fingerprint images...........19 Figure 3.3: Output fingerprint images after using different enhancement algorithms on corrupted (SNR: 5, 10, and $20 \mathrm{~dB}$ ) input fingerprint images. .20 
Figure 3.4: Histogram and Probability Density Function (PDF) representation for with and without fingerprint image enhancement algorithms in case of blurred (using Gaussian Filter- $3 \times 3$ ) input fingerprint images.

Figure 3.5: Histogram and Probability Density Function (PDF) representation for with and without fingerprint image enhancement algorithms in case of blurred (using Gaussian Filter- $5 \times 5$ ) input fingerprint images

Figure 3.6: Histogram and Probability Density Function (PDF) representation for with and without fingerprint image enhancement algorithms in case of blurred (using Gaussian Filter- $7 \times 7$ ) input fingerprint images.

Figure 3.7: Histogram and Probability Density Function (PDF) representation for with and without fingerprint image enhancement algorithms in case of blurred (using Average Filter$3 \times 3$ ) input fingerprint images

Figure 3.8: Histogram and Probability Density Function (PDF) representation for with and without fingerprint image enhancement algorithms in case of blurred (using Average Filter$5 \times 5$ ) input fingerprint images.

Figure 3.9: Histogram and Probability Density Function (PDF) representation for with and without fingerprint image enhancement algorithms in case of blurred (using Average Filter$7 \times 7$ ) input fingerprint images

Figure 3.10: Histogram and Probability Density Function (PDF) representation for with and without fingerprint image enhancement algorithms in case of noisy (SNR $5 \mathrm{~dB}$ ) input fingerprint images. .29

Figure 3.11: Histogram and Probability Density Function (PDF) representation for with and without fingerprint image enhancement algorithms in case of noisy (SNR $10 \mathrm{~dB}$ ) input fingerprint images. .30

Figure 3.12: Histogram and Probability Density Function (PDF) representation for with and without fingerprint image enhancement algorithms in case of noisy (SNR $20 \mathrm{~dB}$ ) input fingerprint images. 


\section{Acknowledgements}

I would like to provide my heartiest appreciation to my advisor: Dr. Chi-Hao Cheng. He has continuously supported me from the beginning of my research. His continuous support and guidance has helped me to be persistent in achieving the outcome of my research. His ingenious ideas and avant-garde approach of scrutinizing the quality of research has helped me to upgrade my research aspect. The most consequential attitude I have learnt from him is his well-disciplined manner which is going to be a great advantage for me in my future.

I would also like to thank Dr. Qihou Zhou and Dr. Yamuna Rajasekhar for being my committee members. Their valuable and insightful comments have helped me to improve the quality of my research. I am really grateful to them for giving me their valuable time and support.

In addition, I would like to thank all the faculty, staff and graduate students of College of Engineering and Computing for supporting me throughout my study here at Miami University and making me experience to study in a better and favorable place.

A special thanks to my family, my parents and my younger sister for supporting me and loving me throughout my life. I would like to give my deepest gratitude and love to my parents for making me who I am today and for believing in me. 


\section{Chapter 1}

\section{Introduction}

Fingerprint can be defined as human fingers' ridge pattern representations [1]. Each person has unique fingerprint thus making fingerprint a useful and convenient biometric identification measure [2-4]. Input fingerprint image quality determines the performance of Automatic Fingerprint Matching (AFM) process and blurred fingerprints can seriously deteriorate the performance of AFM process. The purpose of using enhancement algorithms is to improve the performance of AFM process by enhancing the quality of blurred input fingerprint images. In this study, four fingerprint image enhancement algorithms for blurred fingerprints are considered: Gabor filter, Volterra filter, Laplacian filter and combination of Volterra filter and Gabor filter. Gabor filter is arguably the most popular enhancement method used for fingerprint [5] and Laplacian filter is widely used to sharpen image [3]. Volterra filter is widely used in digital signal processing [3] but not as widely used as Gabor filter nor Laplacian filter.

There are six basic human fingerprint ridge pattern types: arches, tented arches, right loop, left loop, twin loop, and whorl [6-7]. Figure 1.1 shows these six types of ridge patterns.

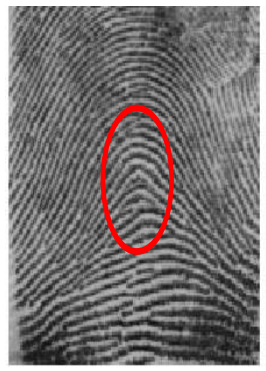

(a)

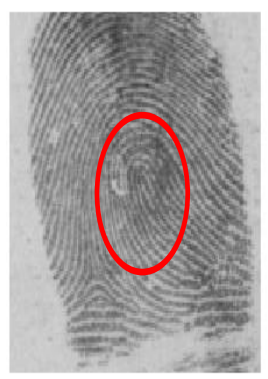

(d)

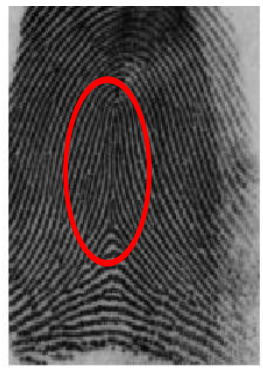

(b)

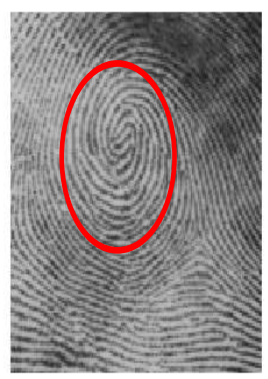

(e)

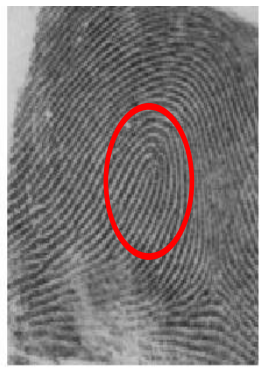

(c)

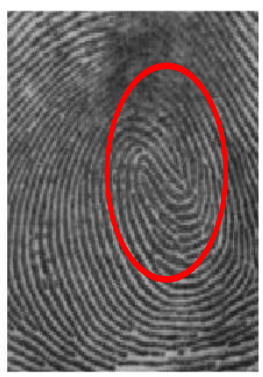

(f)

Figure 1.1: Fingerprint classification: (a) Arch, (b) Tented arch, (c) Right loop, (d) Left loop, (e) Whorl, (f) Twin loop [7].

The six types of ridge patterns shown in Figure 1.1 is explained as following. Arch is one of the very rare $(5 \%)$ type of ridge patterns of human fingerprint. In this type of ridge pattern, a 
significant amount of ridges enters from one side, makes a small blunt spike and exits from the other side. A special type of arch ridge pattern is known as tented arch ridge pattern. The difference between arch and tented arch ridge pattern is, tented arch ridge pattern has a very sharp and long spike compared to arch ridge pattern. Loop is the very common (60-65\%) type of ridge pattern of human fingerprint. In this pattern, a number of ridges enter in the pattern from one side, and after a while it takes a turn and exits from the same side of the pattern. Loop fingerprints are divided into left loop, right loop, and twin loop based on the characteristics of their turning process. Another type of ridge pattern of human fingerprints is defined as whorl (30-35\%). In this patterns, a particular ridge is converted into two other ridges, both of which are known as whorl.

Each particular type of fingerprints shown in Figure 1.1 can be further categorized based on its local ridge characteristics and there are approximately 150 types of local ridge characteristics [8]. The identified local ridge characteristics are bifurcation, island, ridge ending, pore, core, delta, etc. Figure 1.2 shows some local ridge characteristics of fingerprints. Ridge ending and ridge bifurcation are two basic and most used local ridge characteristics which are known as minutiae of fingerprints. In an AFM process, input and reference fingerprint images' fingerprint minutiae are compared using Minutiae Extraction Algorithm (MEA) which computes the similarity score between two fingerprints [9]. Figure 1.3 shows two fingerprint minutiae: ridge ending and ridge bifurcation. The ridge ending can be defined as the point at which a ridge stops continuing its path and bifurcation is the points at which two ridges convert into one particular ridge.

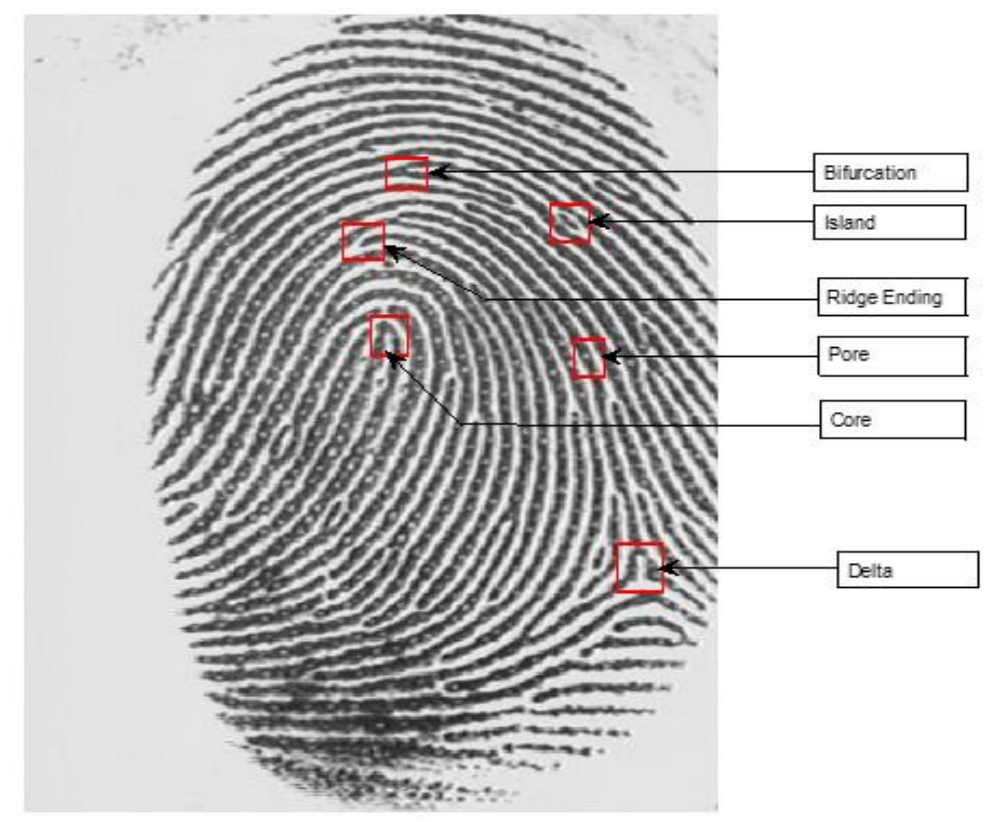

Figure 1.2: Different identified local ridge characteristics. 

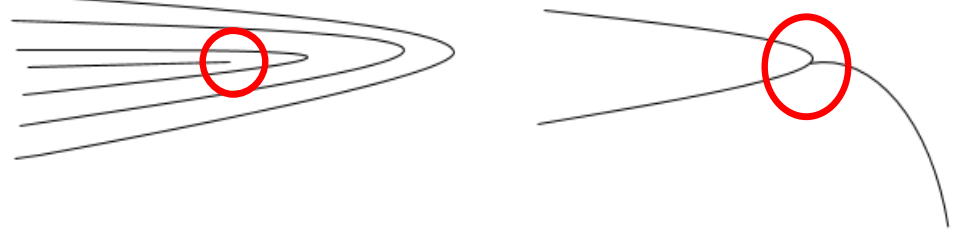

Ridge Ending

Bifurcation

Figure 1.3: Fingerprint minutiae.

Computation of similarity score between input and reference fingerprint images in an AFM process varies based on the characteristics of fingerprint minutiae used in this process. In this study, ridge ending and bifurcation, are used in the determination of similarity score. The performance of AFM process depends on the quality of input fingerprint images. The ridge structures of a good quality fingerprint image are easily observed and a blurred fingerprint's ridge structures are difficult to identify. A good and a blurred fingerprint images are shown in Figure 1.4 (a) and (b) respectively. Sometimes the ridge characteristics of a particular fingerprint image is so corrupted that it cannot be characterized by any local ridge characteristics. This kind of region of a particular fingerprint image is defined as unrecoverable region which is shown in Figure 1.5. In this study, the unrecoverable region of a fingerprint image is ignored. The fingerprint enhancement algorithms are applied only on blurred fingerprint images which do not contain any unrecoverable region. The statistics show that approximately 10 percent of fingerprint images are of poor quality and these poor quality fingerprint images are blurred due to reasons such as inappropriate fingerprint sensor, poor contact between fingers and sensors, wet/sweaty human fingers, poor image resolution, presence of noise, movement of fingertip, etc. [5]. Blurred input fingerprint images cause MEA fails to distinguish between false and genuine minutiae [5] thus deteriorating the accuracy of AFM process [10]. Therefore, in case of blurred fingerprint images an enhancement algorithm must be applied to improve the clarity of the ridge structure. Once the ridge structure becomes significantly visible in fingerprint images, the performance of MEA is uplifted which eventually improve the performance of AFM process.

The rest of the thesis is organized as followings. Theoretical background of different enhancement algorithms as well as evaluation method is described in chapter 2. Experimental results of four enhancement algorithms are provided in chapter 3. Chapter 4 concludes this thesis and summarizes possible future works. 


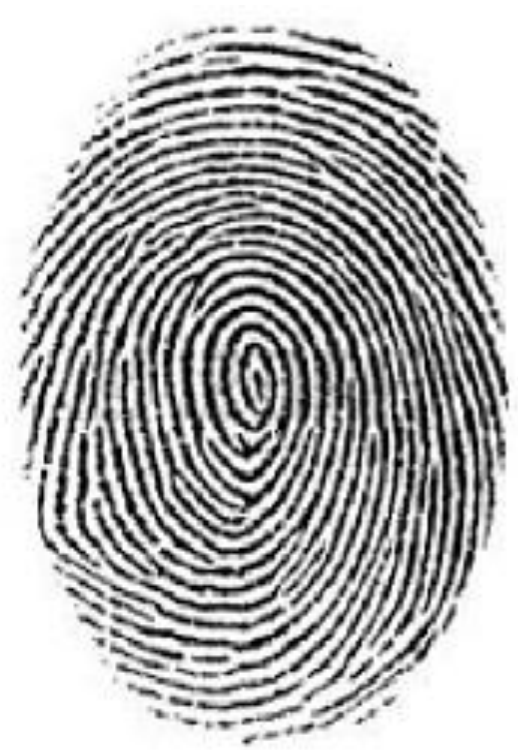

(a)

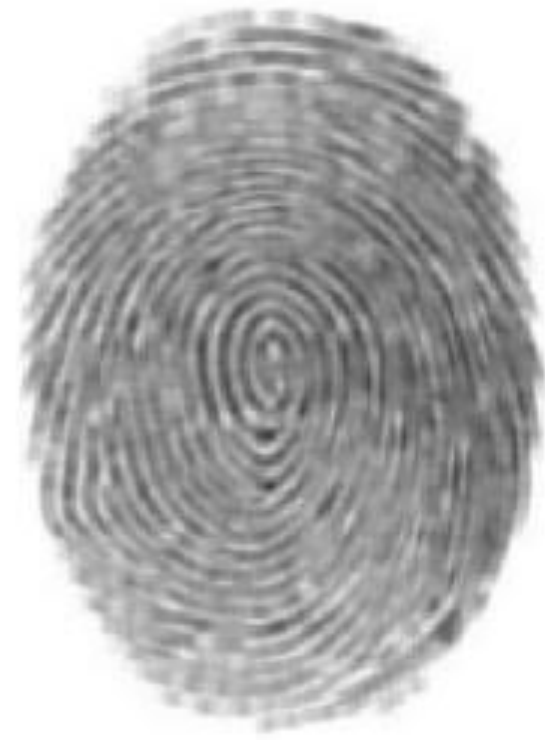

(b)

Figure 1.4: (a) Good quality fingerprint images and (b) blurred fingerprint Images.

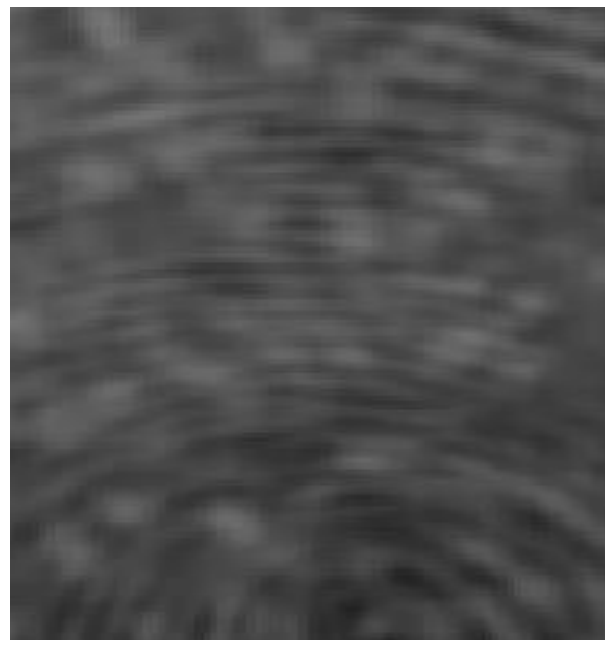

Figure 1.5: Unrecoverable region of fingerprints. 


\section{Chapter 2}

\section{Theoretical Background}

\subsection{Fingerprint Image Enhancement}

Input fingerprint image quality determines the performance of AFM process. Different fingerprint image enhancement algorithms have been used for improving the performance of AFM process by enhancing the quality of blurred input fingerprint images. The basic idea of different fingerprint enhancement algorithms is provided in this chapter.

\subsubsection{Gabor Filter}

Gabor filter is a linear filter used for edge detection. The characteristics of Gabor filter (frequency and orientation representation) is similar to those of a Human Visual System (HVS). Gabor filters are used for extracting features from images. It is the most popular fingerprint image enhancement algorithm.

Several steps are required to use Gabor filter as an enhancement algorithm and they are summarized as following. The original fingerprint image is normalized at the first step [5]. The normalized image is then divided into several small square blocks to find the orientation field estimation of each block [11]. After the orientation field of entire normalized fingerprint image is estimated, the ridge frequency of normalized fingerprint image is then calculated. The orientation field estimation and the value of ridge frequency is used to design a Gabor filter in order to enhance the blurred fingerprint image. In the last step, the normalized image is passed through the Gabor filter. The above procedure will be elaborately described in following paragraphs.

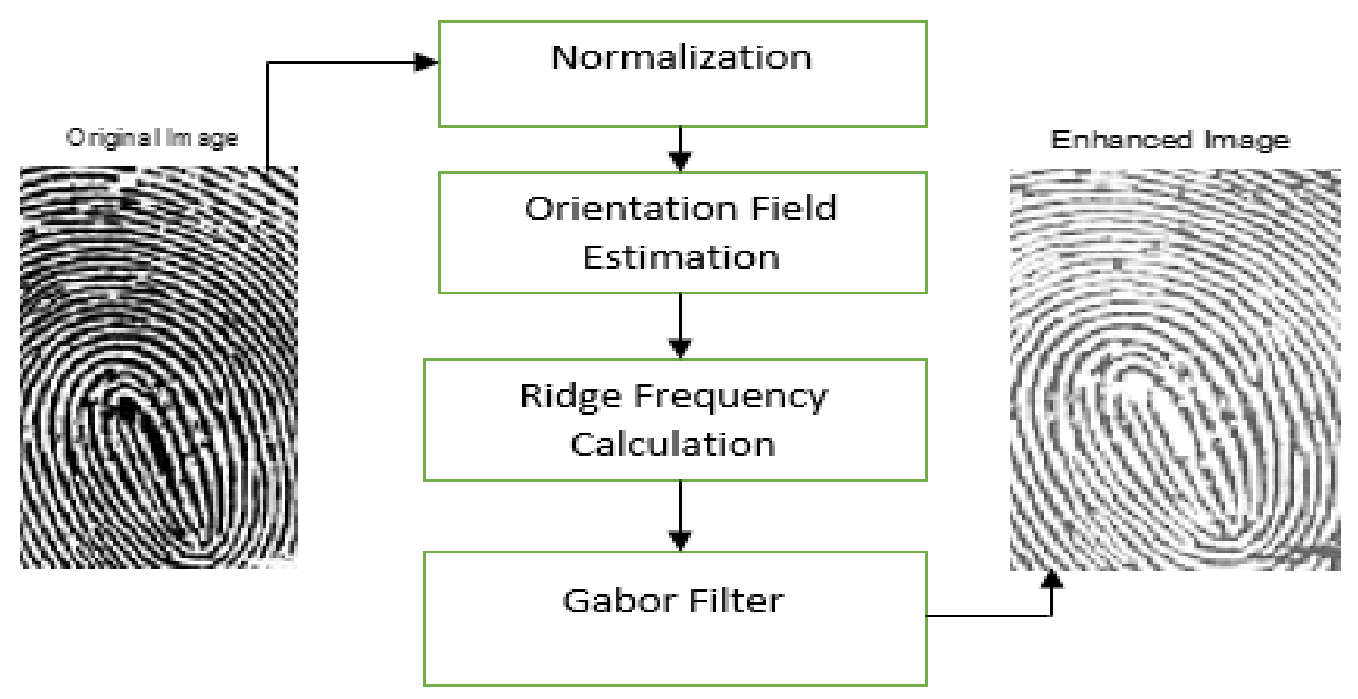

Figure 2.1: Steps of using Gabor filter as fingerprint enhancement algorithm. 
Let $I(i, j)$ be the gray scale value of input fingerprint image at pixel $(i, j), M_{0}$ and $V A R_{0}$ be the estimated mean and variance of image $I$, and $G(i, j)$ be the normalized gray scale value at pixel $(i, j)$. The normalized image is then defined as

$G(i, j)=\left\{\begin{array}{l}M_{0}+\sqrt{\frac{V A R_{0}+(I(i, j)-M)^{2}}{V A R}} \text { if } I(i, j)>M \\ M_{0}-\sqrt{\frac{V A R_{0}+(I(i, j)-M)^{2}}{V A R}} \text { otherwise }\end{array}\right.$

where, $M_{0}$ and $V A R_{0}$ are expected mean and variance which are chosen as 100 for both of the values respectively in this study [5].
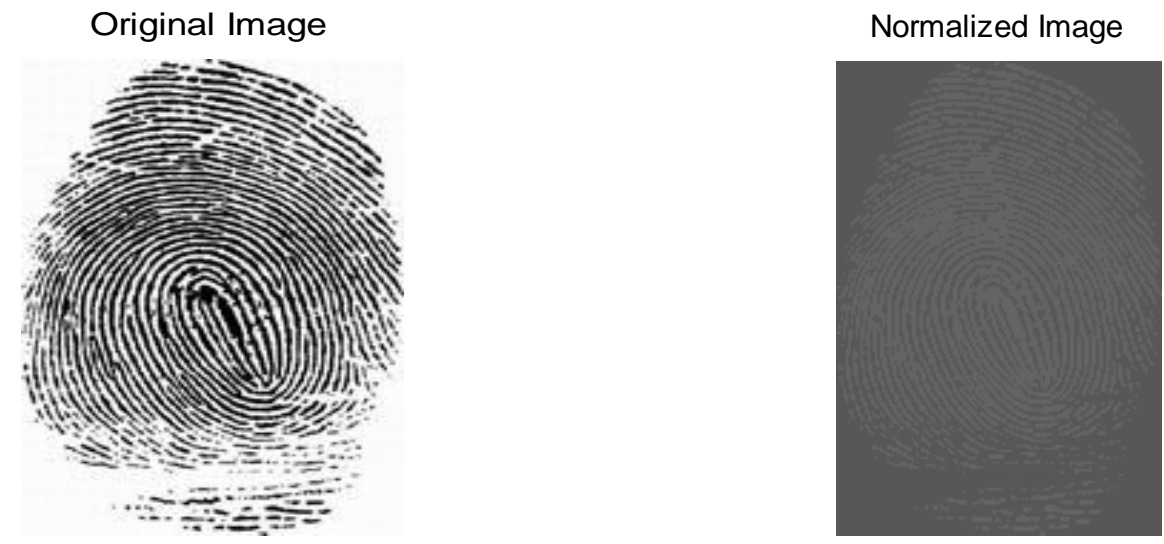

Figure 2.2: Original image and normalized image $\left(\mathrm{M}_{0}=100\right.$ and $\left.\mathrm{VAR}_{0}=100\right)$.

An image and its normalized image are shown in Figure 2.2. In the next step, the normalized image is divided into block size of $w \times w=16 \times 16$. Gradients $\left(G_{x}\right.$ and $\left.G_{y}\right)$ for each pixel of normalized image $G(i, j)$ is computed using a Sobel filter.

$G_{x}(i, j)=\left[\begin{array}{lll}-1 & 0 & +1 \\ -2 & 0 & +2 \\ -1 & 0 & +1\end{array}\right] \circledast \mathrm{G}(\mathrm{i}, \mathrm{j})$
$G_{y}(i, j)=\left[\begin{array}{ccc}-1 & 0 & +1 \\ 0 & 0 & +2 \\ +1 & 0 & +1\end{array}\right] \circledast \mathrm{G}(\mathrm{i}, \mathrm{j})$

where, $\circledast$ is used to define 2-dimensional convolution. Based on the gradients, orientation field estimation $\theta(i, j)$ is calculated using the following equations.

$\mathrm{V} x(i, j)=\sum_{u=i-w / 2}^{i+\frac{w}{2}-1} \sum_{u=j-w / 2}^{j+\frac{w}{2}-1} 2 G_{x}(i, j) G_{y}(i, j)$

$\mathrm{V} y(i, j)=\sum_{u=i-w / 2}^{i+\frac{w}{2}-1} \sum_{u=j-w / 2}^{j+\frac{w}{2}-1} G_{x}(i, j)^{2} G_{y}(i, j)^{2}$ 
$\theta(i, j)=\frac{1}{2} \cdot \tan ^{-1} \frac{V y(i, j)}{V x(i, j)}$

In case of noise, orientation field estimation, $\theta(i, j)$ might be inaccurate. To overcome this issue, a low-pass Gaussian filter is used in this study to achieve appropriate orientation field estimation. For using low pass filter, $\theta(i, j)$ is converted into continuous field by using the following equations.

$O_{x}(i, j)=\cos (2 \theta(i, j))$

$O_{y}(i, j)=\sin (2 \theta(i, j))$

The low pass filtered output of $\theta(i, j)$ along $x$ and $y$ direction is denoted by $O_{x}^{L}(i, j)$ and $O_{y}^{L}(i, j)$ respectively.

$O_{x}^{L}(i, j)=\sum_{p=-\frac{s}{2}}^{\frac{s}{2}-1} \sum_{q=-\frac{s}{2}}^{\frac{s}{2}-1} G L P(p, q) \cdot O_{x}(i-p, j-q)$

$O_{y}^{L}(i, j)=\sum_{p=-\frac{s}{2}}^{\frac{s}{2}-1} \sum_{q=-\frac{s}{2}}^{\frac{s}{2}-1} G L P(p, q) \cdot O_{y}(i-p, j-q)$

where, GLP is a Gaussian low pass filter with unit integral and size $s$ ( $s=5$ in this study). Final orientation field estimation, $O(i, j)$ is achieved using the lowpass filtered output.

$O(i, j)=\frac{1}{2} \times \tan ^{-1} \frac{o_{y}^{L}(i, j)}{O_{x}^{L}(i, j)}$

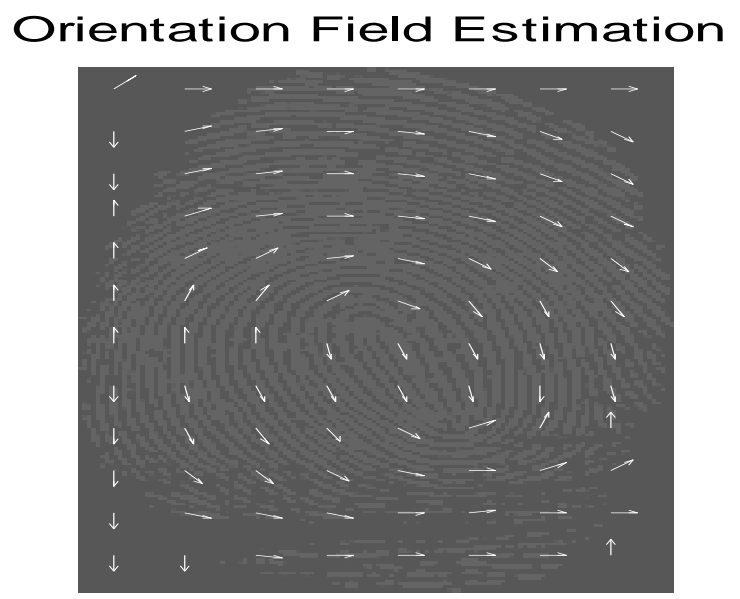

Figure 2.3: Orientation field estimation of input fingerprint image.

Figure 2.3 shows the orientation field estimation $(O(i, j))$ of input fingerprint image. The ridge frequency is calculated based on the normalized image. Normalized image is initially divided into square block of size $w \times w=16 \times 16$. After constructing each square block, another rectangular 
block of size $l \times w=32 \times 16$ is created. For each square block $\mathrm{x}$-signature, $X[k]$, is created as following.

$\mathrm{X}[\mathrm{k}]=\frac{1}{\mathrm{w}} \sum_{\mathrm{m}=0}^{16-1} \mathrm{G}(\mathrm{u}, \mathrm{v}), \mathrm{k}=0,1,2, \ldots \ldots l-1$

$u=i+\left(m-\frac{w}{2}\right) \cos O(i, j)+\left(k-\frac{l}{2}\right) \sin (O(i, j))$

$v=j+\left(m-\frac{w}{2}\right) \sin O(i, j)+\left(k-\frac{l}{2}\right) \cos (O(i, j))$

where, $u$ and $v$ are computed using the orientation field estimation $O(i, j) . G(u, v)$ is the value of normalized image at pixel $u, v . G(u, v)$ is basically the rotated version of $G(i, j)$. X-signature $(X[k]$; where $\mathrm{k}=0,1,2, \ldots \ldots l-1)$ represents a form of sine wave. If the total number of peaks in the $\mathrm{x}$-signature is $T$ and the distance between first and last peak is $d$ then the frequency $f$ is defined as

$f=\frac{d}{T-1}$

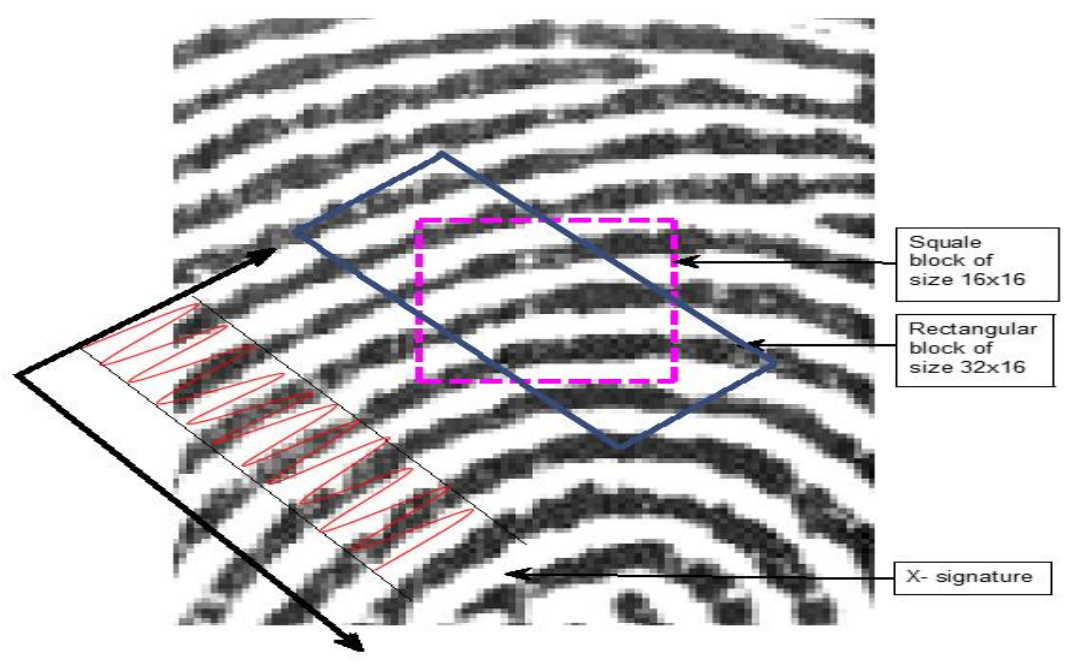

Figure 2.4: Ridge Frequency Calculation.

Figure 2.4 shows the procedure of calculating the ridge frequency. Using normalized image $(G(i, j))$, orientation field estimation $(O(i, j))$ and ridge frequency $(f)$ the output of Gabor filter is achieved. The Gabor filter can be defined as

$H(x, y: O, f)=\exp \left\{-\frac{1}{2}\left[\frac{x_{O}^{2}}{\delta_{x}^{2}}+\frac{y_{O}^{2}}{\delta_{y}^{2}}\right]\right\} \cos \left(2 \pi f x_{O}\right)$

$x_{0}=x \cdot \cos (0)+y \cdot \sin (0)$

$y_{0}=x \cdot \cos (0)+y \cdot \sin (0)$

where, $O$ is the orientation field estimation of the Gabor filter, $f$ is the local ridge frequency computed by using $\mathrm{x}$-signature and $\delta x$ and $\delta y$ are the standard deviations of the Gaussian envelope $(\delta x=\delta y=4$ is used in this study). If the enhanced image after using Gabor filter is denoted by $E(i, j)$, then the enhanced image is defined as 
$E(i, j)=\sum_{u=-\frac{s_{G}}{2}}^{\frac{s_{G}}{2}-1} \sum_{v=-\frac{s_{G}}{2}}^{\frac{s_{G}}{2}-1} h(u, v: O(i, j), f) G(i-u, j-v)$

where, $h$ is the modulation transfer function of Gabor filter, $H$ and the size of Gabor filter is $S_{G}\left(S_{G}=16\right.$ is used in this study). So, in this study, based on the least square orientation field estimation $(O)$ and ridge frequency $(f)$ a Gabor filter of size 16 is implemented and the image is passed through the Gabor filter to be enhanced.

\subsubsection{Laplacian Filter}

Laplacian filter is a linear high-pass filter used to enhance the edge of blurred images [12]. It is a two dimensional isotropic filter. Laplacian filter enhances the images by calculating the second derivative of an image. The Laplacian operator $\Delta^{2} I(x, y)$ of an image $I$ is

$$
\Delta^{2} I(x, y)=\frac{\partial^{2} \mathrm{I}}{\partial \mathrm{x}^{2}}+\frac{\partial^{2} \mathrm{I}}{\partial \mathrm{y}^{2}}
$$

where, $\frac{\partial^{2} \mathrm{I}}{\partial \mathrm{x}^{2}}$ and $\frac{\partial^{2} \mathrm{I}}{\partial \mathrm{y}^{2}}$ are the second derivatives of image $I$ along $x$ and $y$ direction respectively.

$$
\begin{aligned}
& \frac{\partial^{2} \mathrm{I}}{\partial \mathrm{x}^{2}}=I(x+1, y)+I(x-1, y)-2 I(x, y) \\
& \frac{\partial^{2} \mathrm{I}}{\partial \mathrm{y}^{2}}=I(x, y+1)+I(x, y-1)-2 I(x, y)
\end{aligned}
$$

Substituting the values of $\frac{\partial^{2} \mathrm{I}}{\partial \mathrm{x}^{2}}$ and $\frac{\partial^{2} \mathrm{I}}{\partial \mathrm{y}^{2}}$ in the equation of $\Delta^{2} I(x, y)$, we get

$$
\Delta^{2} I(x, y)=I(x+1, y)+I(x-1, y)+I(x, y+1)+I(x, y-1)-4 I(x, y)
$$

The blurred image is enhanced by adding the Laplacian filter output multiplied by a constant to itself as shown below.

$E(x, y)=I(x, y)+\alpha \Delta^{2} I(x, y)$

where, $E(x, y)$ is the enhanced image, $\alpha$ is a constant and $\Delta^{2} I(x, y)$ is the Laplacian operator of image I. In this article, the value of $\alpha$ is chosen as -1 .

Basically the Laplacian filter captures intensity change of a blurred image and the output of Laplacian filter is added back to the original image to enhance its edge. Laplacian filter is widely used for edge enhancement due to its computational simplicity. As a high pass filter, it enhances the high frequency region of an image which is known as edge of the image [13]. A particular problem of Laplacian filter is that it also amplifies noise in the darker region while enhancing the edges. This phenomenon weakens the performance of Laplacian filter as a fingerprint enhancement algorithm. 


\subsubsection{Volterra Filter}

The basic characteristic of an ideal image enhancement algorithm consists of the ability to enhance in the bright region of an image while mitigating the enhancement process in the dark region. This ingenious idea indicates an enhancement algorithm which is the multiplication of a high pass filter and an average pixel intensity of an image. The Volterra filter is a non-linear filter which is based on an input output relation expressed in a Volterra series. It represents the most natural extension of linear filters [3]. The Volterra filter works on the products of input samples and a onedimensional $2^{\text {nd }}$-order Volterra filter is given below [14]:

$y(n)=\sum_{k 1=-\infty}^{\infty} h_{1}\left(k_{1}\right) x\left(n-k_{1}\right)+\sum_{k 1=-\infty}^{\infty} \sum_{k 2=-\infty}^{\infty} h_{2}\left(k_{1}, k_{2}\right) x\left(n-k_{1}\right) x\left(n-k_{2}\right)$

where $x(n)$ is input signal, $y(n)$ is output signal, $h_{1}\left(k_{1}\right)$ is the first order coefficient and $h_{2}\left(k_{1}, k_{2}\right)$ is the second order coefficients. It is worthy of mention that the first summation on the right of Eq. (2.25) is a standard linear finite impulse response (FIR) filter. An example of the $2^{\text {nd }}$-oder Volterra filter is the Teager's operator as shown below:

$y(n)=x^{2}(n)-x(n-1) \cdot x(n+1)$

It is introduced by Kaiser to calculate the energy $y(n)$ of a one dimensional signal $x(n)$ [15]. Since the complexity of a Volterra filter grows exponentially as its order increases, in this study, only a $2^{\text {nd }}$-order Volterra filter is considered and the Volterra filter under consideration has only second order term. The Volterra filter used in this study is given below:

$y\left(n_{1}, n_{2}\right)=C 1 x^{2}\left(n_{1}, n_{2}\right)-C 2 x\left(n_{1}+1, n_{2}+1\right) \cdot x\left(n_{1}-1, n_{2}-1\right)-C 3 x\left(n_{1}+1, n_{2}-\right.$ 1) $\cdot x\left(n_{1}-1, n_{2}+1\right)-C 4 x\left(n_{1}+1, n_{2}\right) \cdot x\left(n_{1}-1, n_{2}\right)-C 5 x\left(n_{1}, n_{2}+1\right) \cdot x\left(n_{1}, n_{2}-\right.$ 1)

where, $C 1, C 2, C 3, C 4$ and $C 5$ are the filter coefficients of the Volterra filter. By checking Eq. (2.27), people might notice its similarity to Eq. (2.26) and its missing the $1^{\text {st }}$-order term. The Eq. (2.27) is a Volterra filter consisting of only $2^{\text {nd }}$-order term to capture the intensity change of an image. Therefore, the filter coefficients should be designed in such way that when there is no intensity change, the output of Volterra filter equals to zero. Just like the Laplacian filter, the output of Volterra filter is added back to the blurred image to enhance it.

Figure 2.5 shows usage of Volterra filter as a fingerprint enhancement tool. The input blurred fingerprint image is sent through the Volterra filter. The output of Volterra filter is multiplied by a constant ( $\rho=0.5$ in this study) and added back to the input blurred fingerprint image in order to provide an enhanced fingerprint image.

In this study, four fingerprint enhancement methods: Gabor filter, Laplacian filter, Volterra filter and combination of Volterra filter and Gabor filter are investigated. The method used to evaluate their performances is described in section 2.2. 


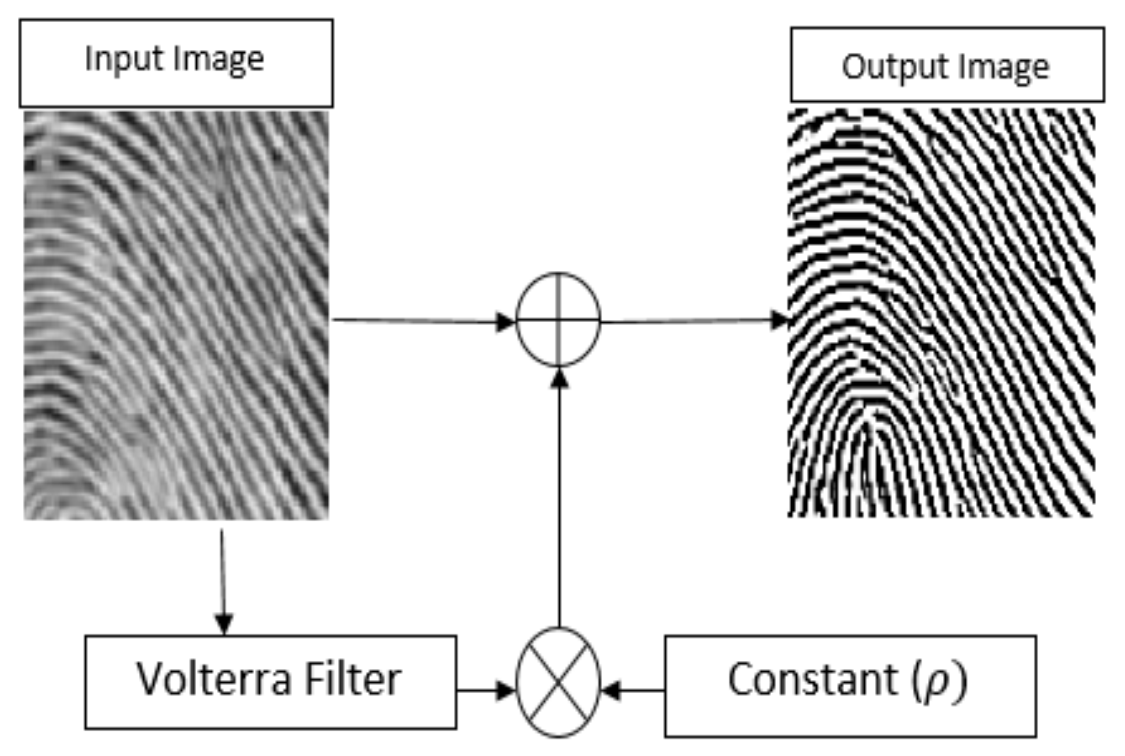

Figure 2.5: Block diagram of Volterra filter as a fingerprint enhancement algorithm.

\subsection{Evaluation Method}

If a fingerprint enhancement method functions as expected, it should improve the performance of a fingerprint matching system. If a fingerprint enhancement method works as expected, the similarity score between enhanced image and correct reference image should be higher than the similarity score between unenhanced image and correct reference images. Moreover, the similarity score between enhanced image and wrong reference image should be lower than the similarity score between unenhanced image and wrong reference images. In this study, the similarity score is used as a method to evaluate fingerprint enhancement methods and the generation of similarity score is described in this section.

Another approach was initially used in this study to evaluate the performance of different enhancement algorithms. In this approach, the Quality Factor (QF) of a particular fingerprint image enhanced by different enhancement algorithms was computed using the power spectrum and entropy of that particular fingerprint image. The approach was experimented on 50 poor quality fingerprint images. It was observed that the values of $\mathrm{QF}$ fail to provide a sound comparison about the performance of different enhancement algorithms as the different between QF of images enhanced by different algorithms is very small thus making it inappropriate to reach a solid conclusion about the performance of different enhancement algorithms. In order to make a sound judgement about the performance of different enhancement algorithms, this approach was replaced by the evaluation method just mentioned: Computation of similarity score between input and reference fingerprint images and we will describe this approach below. The theoretical background of the initial evaluation approach (evaluation method using QF) is described in appendix $\mathrm{A}$ and the experimental results of this approach as a form of a table is provided in appendix B. 
In our study, twenty-one good quality fingerprint images from FVC database 2004 [16] are blurred using Gaussian filter or Average filter to simulate blurred fingerprint images people might encounter in practice. In order to observe the performance of different enhancement algorithms in case of noisy fingerprint images, twenty-one good quality fingerprint images are also corrupted by Gaussian noise where the SNR is set at 5, 10, and $20 \mathrm{~dB}$. Fingerprint image enhancement algorithms are then applied on these blurred and corrupted fingerprint images to improve the performance of AFM process. The fingerprint image enhancement algorithms are analyzed and compared to develop a tangible idea about the characteristics and reliability of these algorithms. Figure 2.6 shows the block diagram of evaluation method for four different enhancement algorithms.

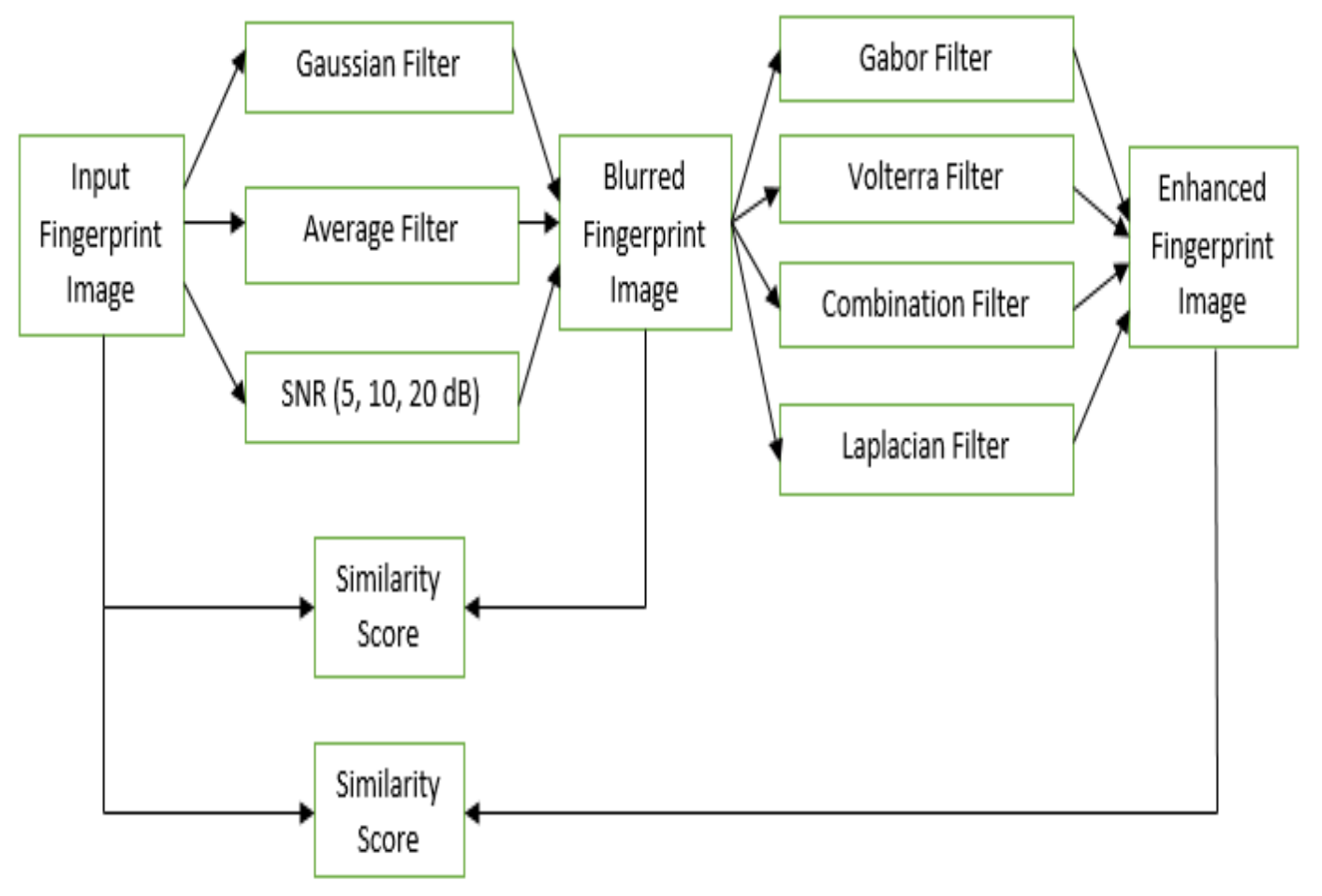

Figure 2.6: A block diagram representing the evaluation method of different enhancement algorithms.

The similarity score can be defined as the square root of a ratio between the square of number of matched minutiae between input and reference fingerprint images and the product of minutiae of these two fingerprint images $[3,7,9,17]$. We developed our programs to calculate the similarity score between two fingerprints using programs developed by V. K. Alilou as a starting point [18]. Two minutiae obtained from input and reference fingerprint images are considered to be matched if the distance between those minutiae in terms of position and direction are lower than the threshold values determined for position and direction. In order to compare the minutiae between two fingerprints, minutiae are extracted from both fingerprints individually using MEA. The most 
commonly used MEA is based on Crossing Number (CN) [19-21]. In our study, two most prominent minutiae: ridge ending and bifurcation are considered while extracting minutiae from fingerprints and minutiae extraction is performed on binary fingerprint images. The first step of MEA is to convert the gray scale image of fingerprint into binary image. Binary image can be defined as an image having only two possible values 0 or 1 for each pixel. Then thinning is performed on binary image. Thinning can be defined as a morphological operation to remove the selected foreground pixels and thins the objects of an image to lines [22-23]. Thinning is based on hit and miss morphological operation. The minutiae are extracted by using a $3 \times 3$ window which scans the local neighborhood of each pixel of thinned binary fingerprint image in order to find the values of $\mathrm{CN}$ for that particular pixel [24]. $\mathrm{CN}$ is computed based on the difference between two neighboring pixels and the half of the sum of total differences is defined as $\mathrm{CN}$.

$C N=\frac{1}{2} \sum_{i=1}^{8}\left|p_{i}-p_{i+1}\right|, \quad p_{1}=p_{9}$

where, $p_{i}(i=1,2, \ldots .9)$ is the $i-t h$ pixel in a thinned binary fingerprint image for a $3 \times 3$ window. In this study, two most prominent minutiae: ridge ending and bifurcation are extracted from thinned binary fingerprint images. The value of each pixels of ridge ending and bifurcation of a thinned binary image for a $3 \times 3$ window can be represented as follows:

\begin{tabular}{|l|l|l|}
\hline 1 & 0 & 1 \\
\hline 1 & $R_{(x, y)}$ & 1 \\
\hline 1 & 1 & 1 \\
\hline
\end{tabular}

(a)

\begin{tabular}{|l|l|l|}
\hline 1 & 0 & 1 \\
\hline 1 & $B_{(x, y)}$ & 0 \\
\hline 1 & 0 & 1 \\
\hline
\end{tabular}

(b)|

Figure 2.7: Pixel values of a $3 \times 3$ window for (a) ridge ending and (b) bifurcation of a thinned binary fingerprint image.

Figure 2.7 shows the pixel representation of $3 \times 3$ window in case of ridge ending and bifurcation of a thinned binary fingerprint image where, $R_{(x, y)}$ and $B_{(x, y)}$ are defined as ridge ending and bifurcation points at $x, y$. The $\mathrm{CN}$ for ridge ending and bifurcation are 1 and 3 respectively which are computed based on Eq. (2.28) and pixel representations of $3 \times 3$ window for each cases.

In our study, a minutia is extracted from thinned binary fingerprint image when the value of $\mathrm{CN}$ for a particular pixel is either 1 or 3 [25]. Figure 2.8 shows the procedure of extracting minutiae from a fingerprint using MEA. 


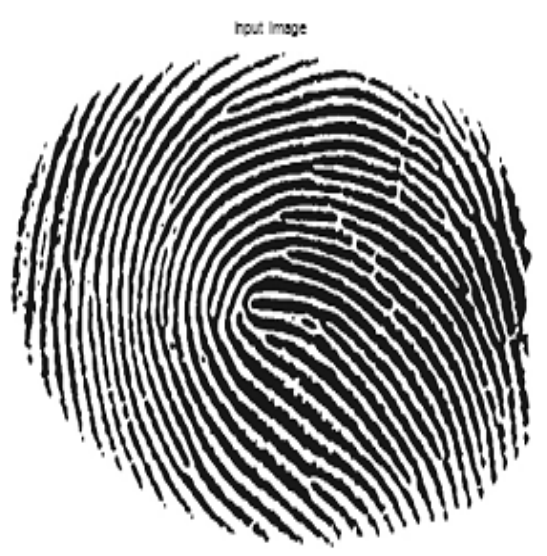

(a)

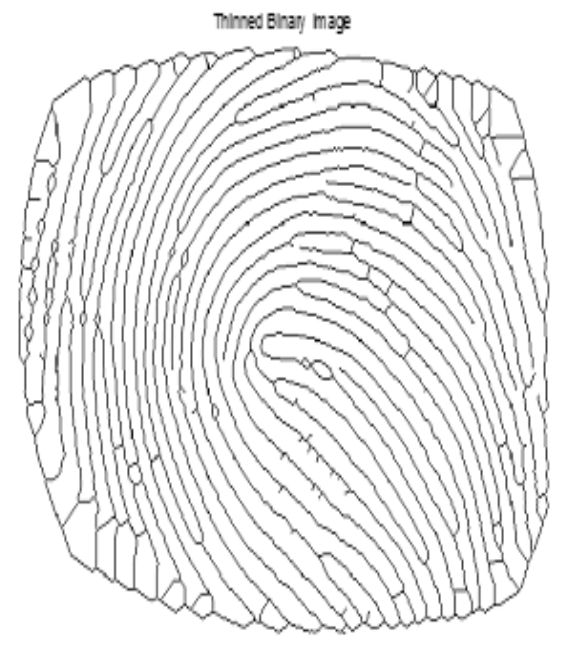

(c)

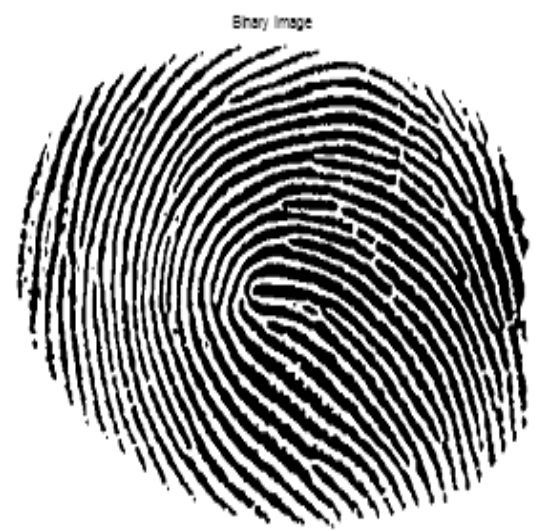

(b)

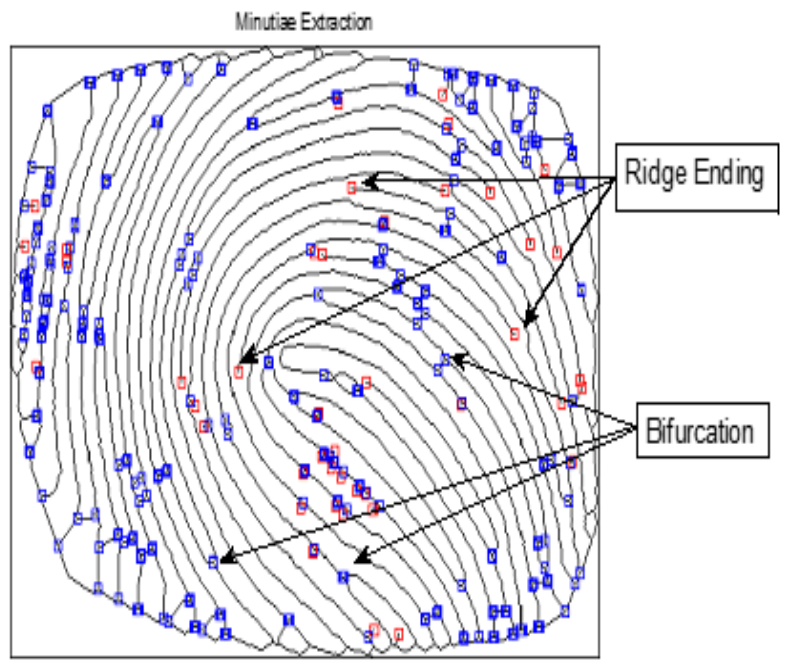

(d)

Figure 2.8: Minutiae extraction: (a) Input gray scale image, (b) Binary image, (c) Thinned binary image and (d) Extracted minutiae.

Once minutiae are extracted similarity score can be obtained using the following computations. If the minutiae sets obtained from MEA for input and reference fingerprint images are $I=$ $\{m 1, m 2, m 3, \ldots ., m i\}$ and $J=\{m 1, m 2, m 3, \ldots \ldots, m j\}$ then the difference in their position $(d p)$ and direction $(d d)$ are determined by using the following formulae

$$
\begin{aligned}
& d p=\sqrt{\left(x_{i}-x_{j}\right)^{2}+\left(y_{i}-y_{j}\right)^{2}} \\
& d d=\min \left(\left|\theta_{i}-\theta_{j}\right|, 360-\left|\theta_{i}-\theta_{j}\right|\right)
\end{aligned}
$$


where, $x_{i}, x_{j}$ and $y_{i}, y_{j}$ are the positions of minutiae from minutiae sets $I$ and $J$ along $x$ direction and $y$ direction respectively and $\theta_{i}, \theta_{j}$ are the angles of minutiae from minutiae sets $I$ and $J$ respectively.

If the threshold values for both position and direction are $r_{0}$ and $\theta_{0}$ respectively $\left(r_{0}=8\right.$ and $\theta_{0}=7$ are used in this study) then a particular minutia is considered to be matched only if they satisfy the conditions i.e. $d p \leq r_{0}$ and $d d \leq \theta_{0}$. The value of SS can be determined by

$\mathrm{SS}=\sqrt{\frac{n^{2}}{i * j}}$

where, $n$ is defined as the number of total matched minutiae and $i$ and $j$ are the number of total extracted minutiae from input and reference fingerprint images respectively. 


\section{Chapter 3}

\section{Experimental Results}

The ultimate purpose of fingerprint enhancement algorithms is to enhance performance of AFM process via improving the quality of fingerprint images. In this study, 21 good quality fingerprint images are taken from FVC database 2004. These images are then blurred by Gaussian filter (size: $3 \times 3,5 \times 5,7 \times 7)$ and average filter (size: $3 \times 3,5 \times 5,7 \times 7)$. Gaussian noise is added to these good quality images and SNR is set at 5, 10 and $20 \mathrm{~dB}$ to observe the performance of enhancement algorithms in case of noisy input fingerprint images. Gabor filter, Volterra filter, Laplacian filter and combination of Volterra filter and Gabor filter are four methods under investigation in this study.

As described in Chapter 2, the Volterra filter used in this study is defined in Eq. (2.27) has five coefficients and we would like determine their values the first. To make sure that the Volterra filter's output is zero when the input image has constant intensity, the following relation needs to satisfy:

$$
C 1-C 2-C 3-C 4-C 5=0
$$

Five sets of coefficients are proposed to emphasize intensity change in different orientation and their values can be found in Table 3.1. Five Volterra filters, each of which has a different set of coefficients are used to process 21 fingerprint images blurred by a Gaussian filter $($ size: $3 \times 3)$ and then calculated the SS between the filter output images and original pre-blurred images. The results are included in Table 3.1. Table 3.1 shows that, out of five combinations, first and third combination provide the highest similarity score. As a result, it would be rational to use either first or third set of Volterra filter coefficients. In this study, the first set of Volterra filter coefficients which is also the Volterra filter coefficients used by other researchers [3] is used.

\section{Table 3.1}

Mean Values of Similarity Score between Reference and Enhanced (Processing the Blurred (using a Gaussian Filter (size: $3 \times 3$ ) Fingerprint Images with Different Combination of Volterra Filter Coefficients) Fingerprint Images.

\begin{tabular}{|c|c|c|c|c|c|c|}
\hline $\begin{array}{c}\text { Volterra Filter } \\
\text { Coefficients }\end{array}$ & $\mathrm{C} 1$ & 3 & 4 & 3 & 2 & 2 \\
\cline { 2 - 7 } & $\mathrm{C} 2$ & -0.5 & -1 & -1 & -1 & 0 \\
\cline { 2 - 7 } & $\mathrm{C} 3$ & -0.5 & -1 & -1 & -1 & 0 \\
\cline { 2 - 7 } & $\mathrm{C} 4$ & -1 & -1 & -0.5 & 0 & -1 \\
\cline { 2 - 8 } & $\mathrm{C} 5$ & -1 & -1 & -0.5 & 0 & -1 \\
\hline \multicolumn{2}{|l}{ Similarity Score } & 0.4703 & 0.4205 & 0.4703 & 0.3281 & 0.2814 \\
\hline
\end{tabular}


The performance of image enhancement algorithms can be evaluated by visually comparing original, blurred, and processed images. Figures 3.1-3.3 shows the output of different filters processing upon different blurred/corrupted images along the original and blurred/corrupted images. Results show Volterra filter gives the most promising output among all image enhancement algorithms.

Although a visual check provides useful insights about the filter performance, in order to make more objective evaluation, a quantitate performances evaluation is necessary. To achieve this goal, the similarity score between the reference fingerprints and blurred/corrupted fingerprints are calculated the first. Then, blurred/corrupted fingerprint are enhanced using different enhancement algorithms and similarity score between enhanced fingerprints and original fingerprints are calculated. Twenty-one images are used in this study and the average of similarity score for each cases are provided in Table 3.2.
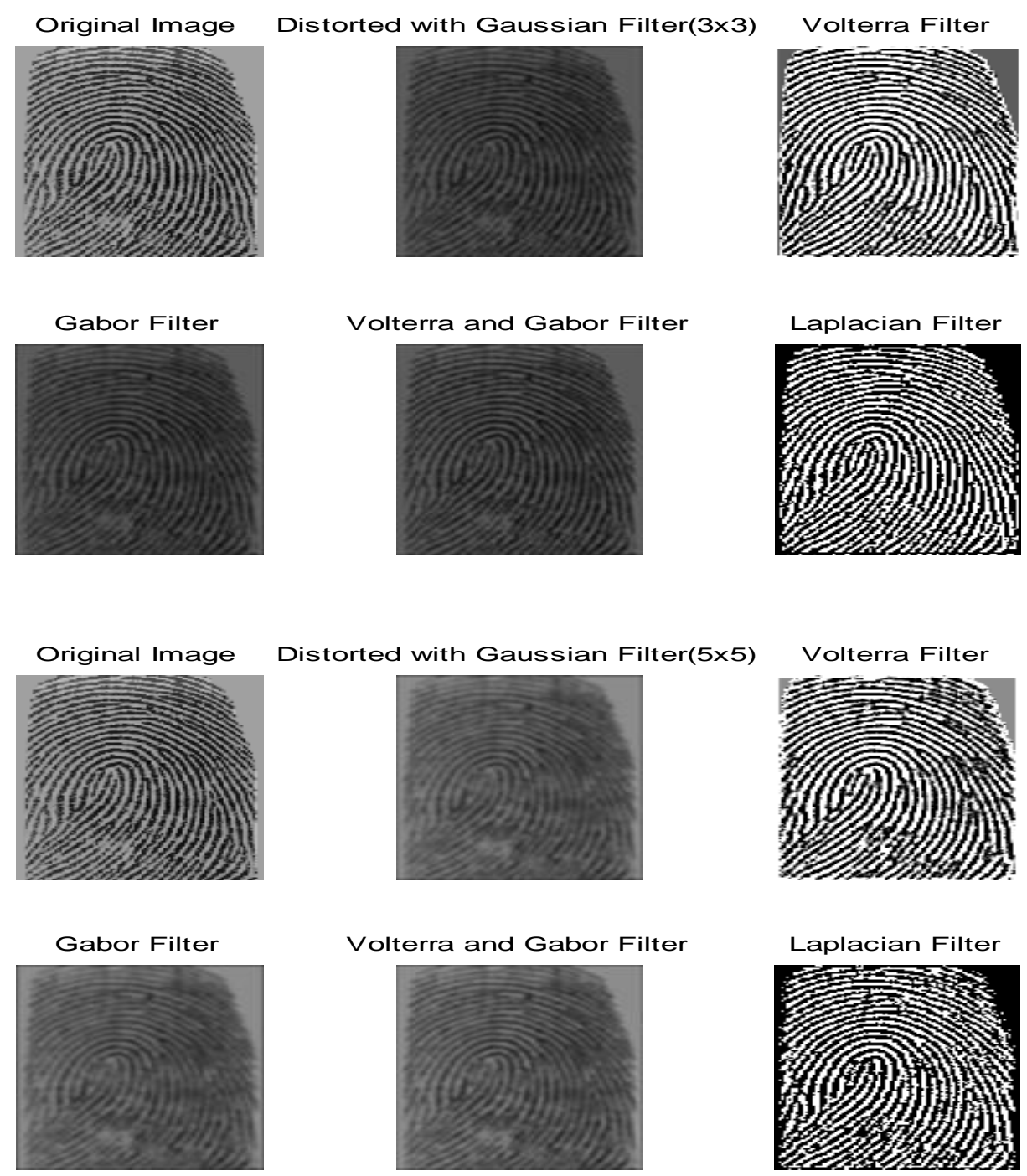


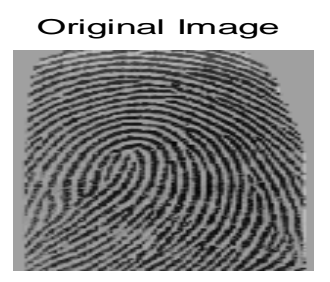

Gabor Filter

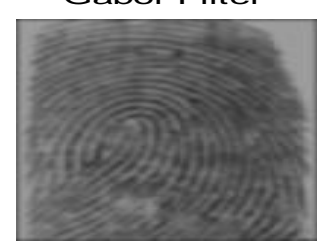

Distorted with Gaussian Filter( $7 \times 7)$

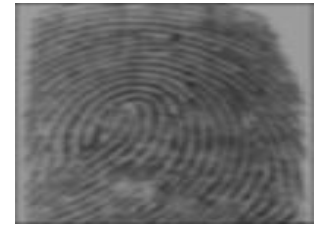

Volterra and Gabor Filter

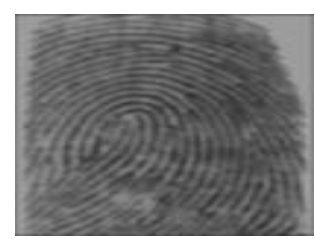

Volterra Filter

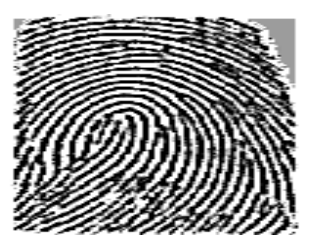

Laplacian Filter

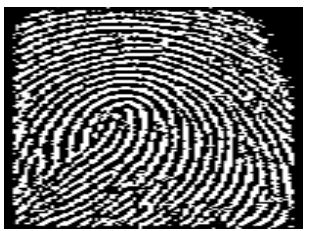

Figure 3.1: Output fingerprint images after using different enhancement algorithms on blurred (using Gaussian filter (size: $3 \times 3,5 \times 5,7 \times 7)$ ) input fingerprint images.
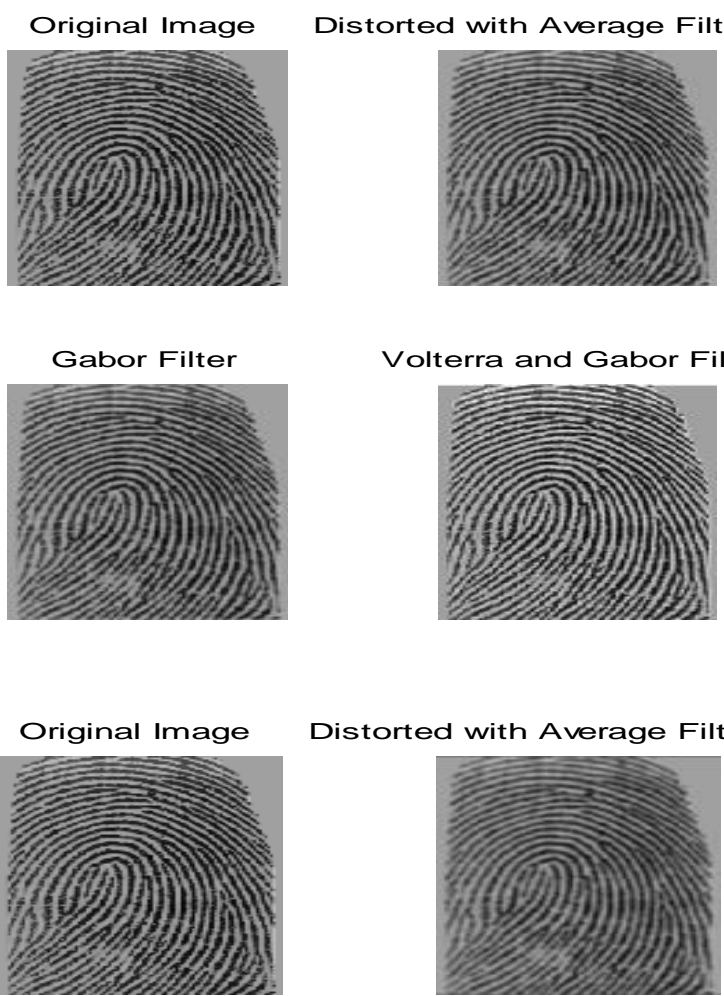

Gabor Filter
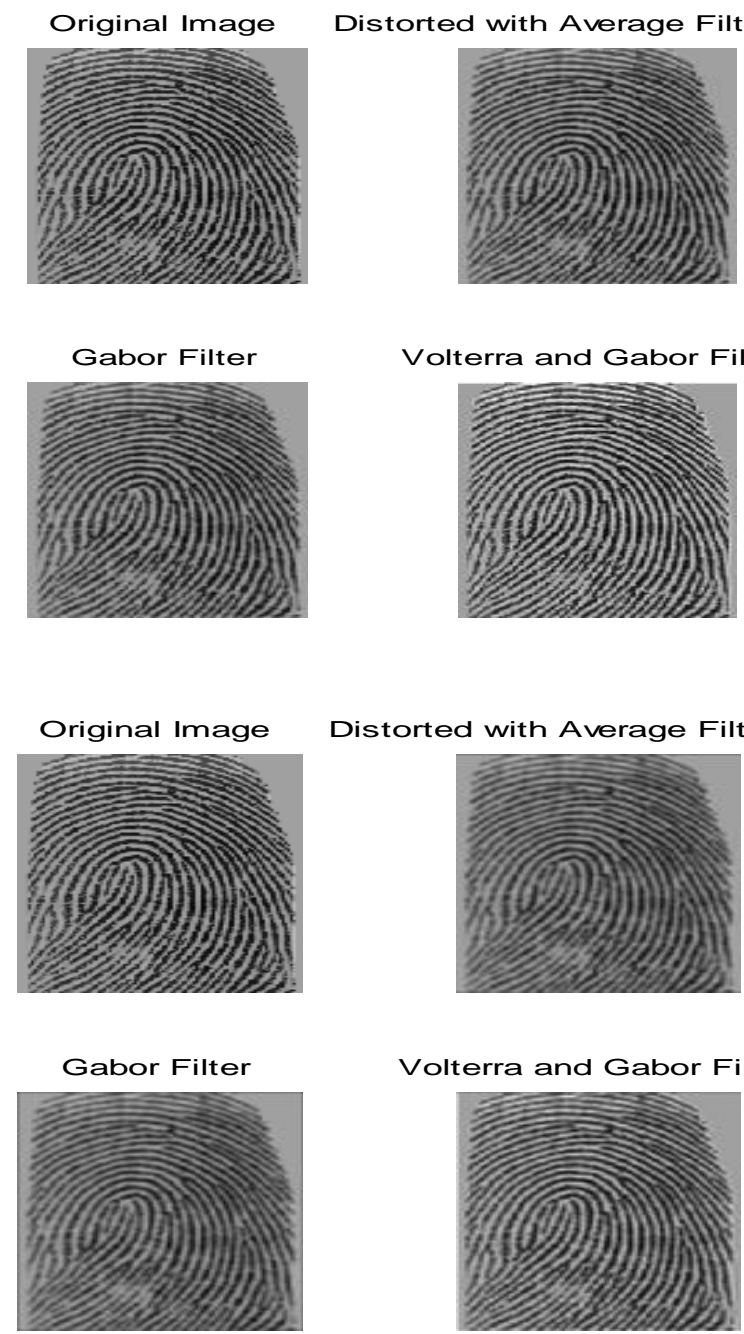

Volterra and Gabor Filter
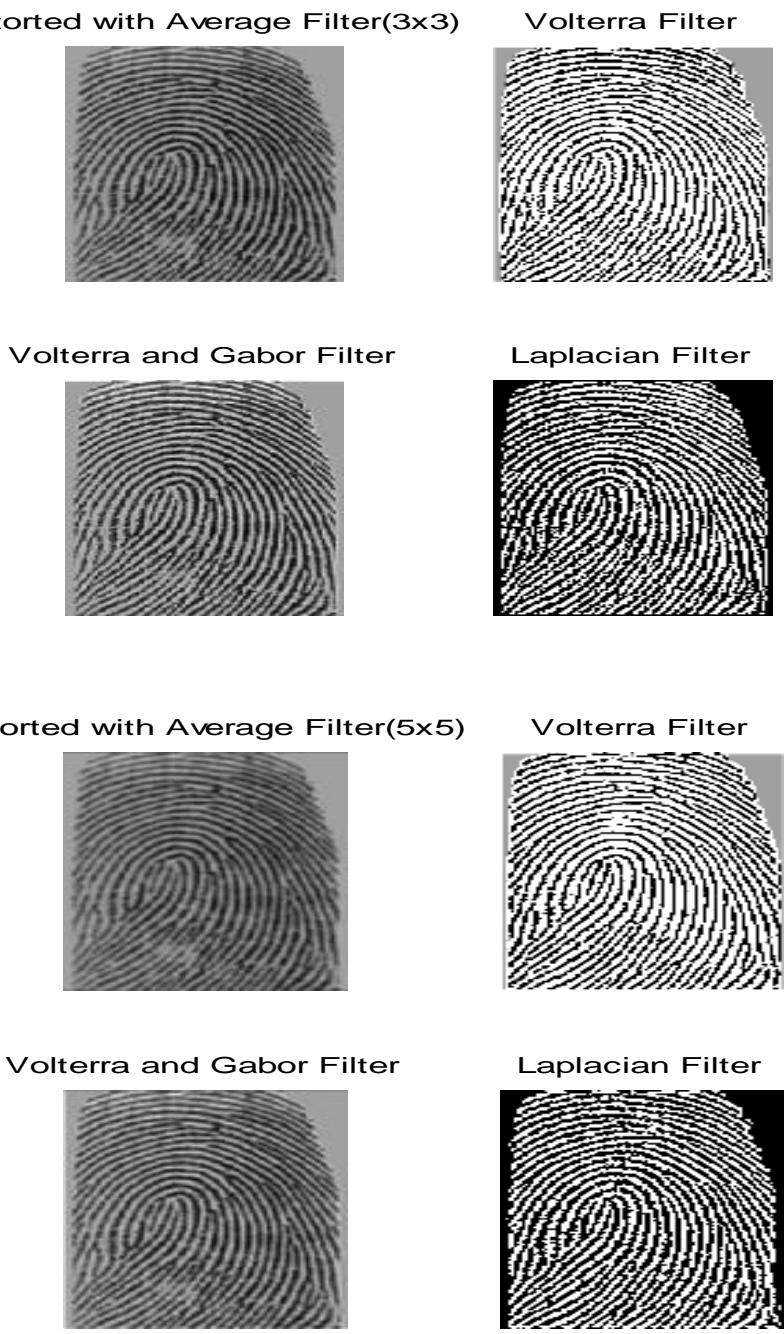

Volterra Filter

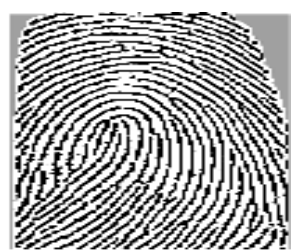

Laplacian Filter

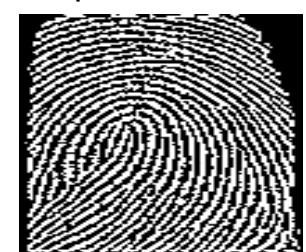



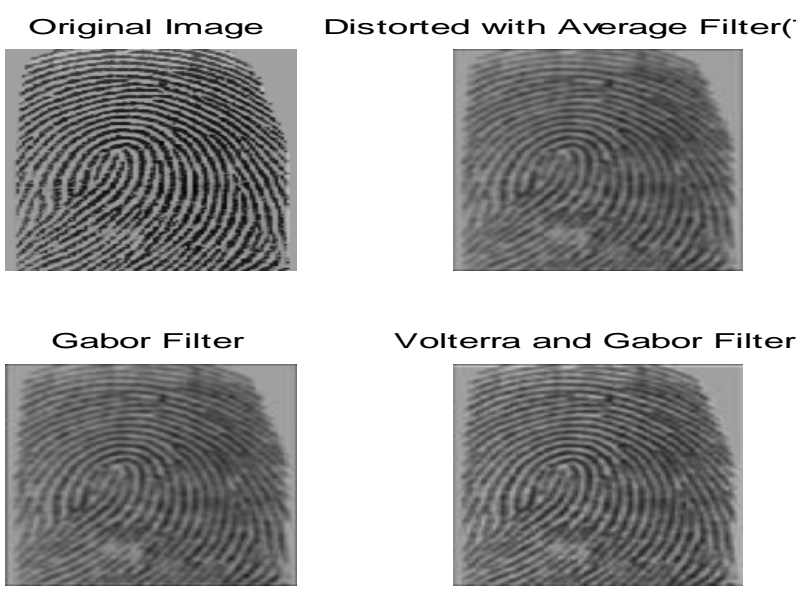
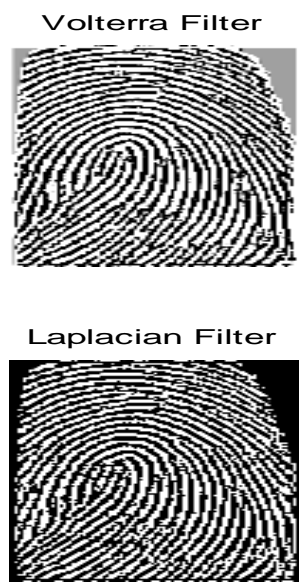

Figure 3.2: Output fingerprint images after using different enhancement algorithms on blurred (using Average filter (size: $3 \times 3,5 \times 5,7 \times 7)$ ) input fingerprint images.

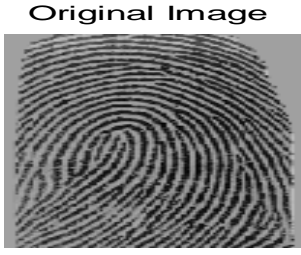

Gabor Filter

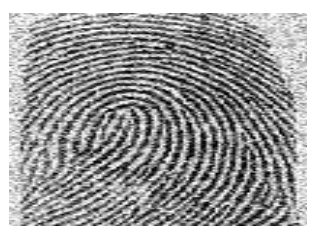

Original Image

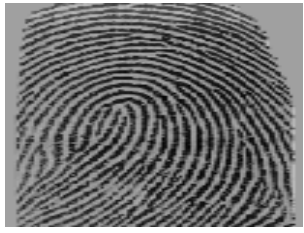

Gabor Filter

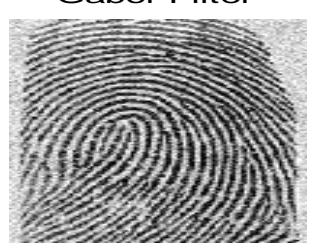

Distorted with SNR $5 \mathrm{~dB}$

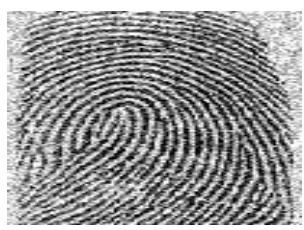

Volterra and Gabor Filter

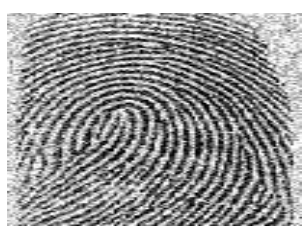

Distorted with SNR $10 \mathrm{~dB}$

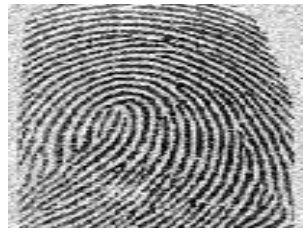

Volterra and Gabor Filter

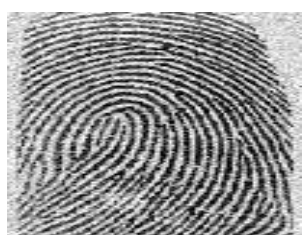

Volterra Filter

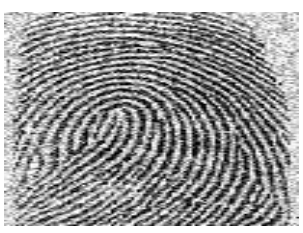

Laplacian Filter

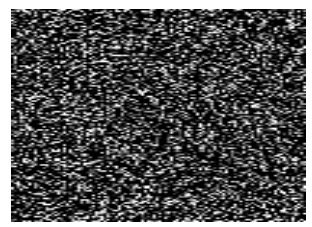

Volterra Filter

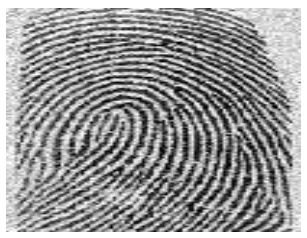

Laplacian Filter

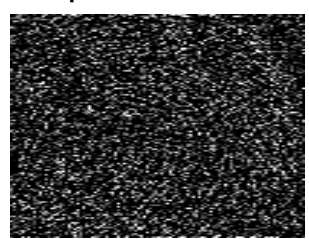



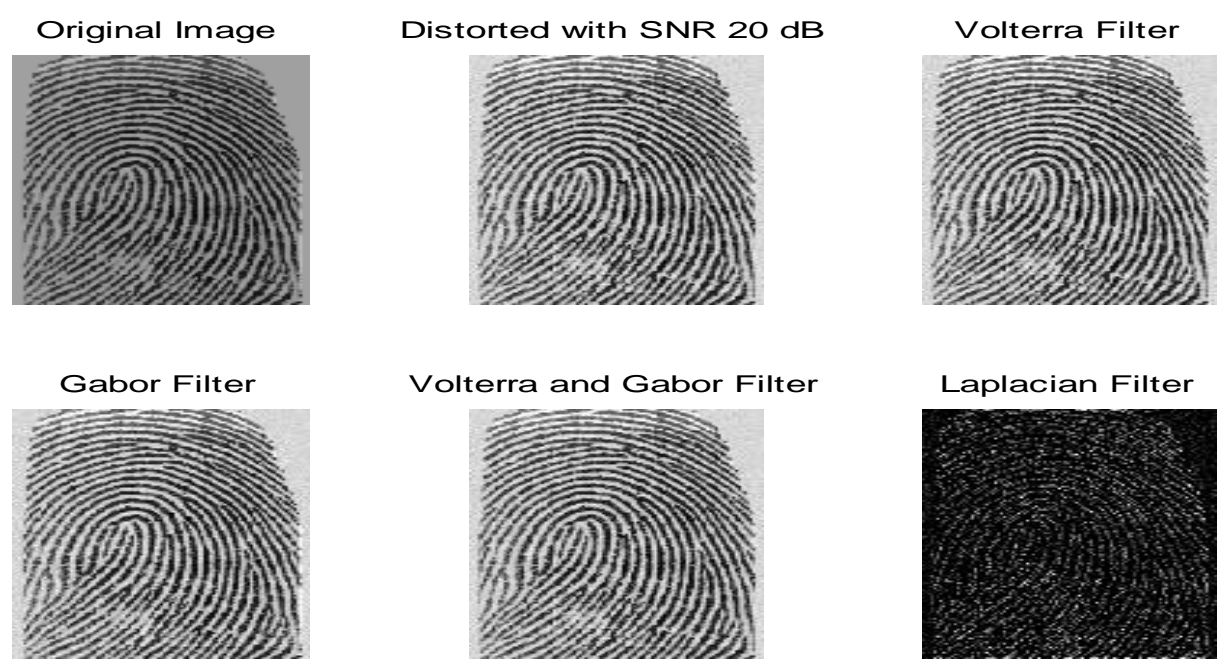

Figure 3.3: Output fingerprint images after using different enhancement algorithms on corrupted (SNR: 5, 10, and $20 \mathrm{~dB}$ ) input fingerprint images.

Table 3.2

Mean Values of Similarity Score between Reference and Unenhanced (Blurred by Gaussian Filter (size: $3 \times 3,5 \times 5,7 \times 7$ ), Average Filter (size: $3 \times 3,5 \times 5,7 \times 7)$ and Corrupted by noise with SNR 5, 10 and $20 \mathrm{~dB}$ ) Matched Fingerprint Images and Mean Values of Similarity

Score between Reference and Enhanced (using Different Enhancement Algorithms on Blurred/Corrupted Images) Matched Fingerprint Images.

\begin{tabular}{|c|c|c|c|c|c|}
\hline \multirow{2}{*}{$\begin{array}{c}\text { Blurred or } \\
\text { Distorted Input } \\
\text { Fingerprint } \\
\text { Images }\end{array}$} & $\begin{array}{c}\text { Without } \\
\text { Enhancement }\end{array}$ & \multicolumn{3}{|c|}{ Similarity Score } \\
\cline { 3 - 6 } & & $\begin{array}{c}\text { Gabor } \\
\text { Filter }\end{array}$ & $\begin{array}{c}\text { Volterra } \\
\text { Filter }\end{array}$ & $\begin{array}{c}\text { Combination } \\
\text { Filter }\end{array}$ & $\begin{array}{c}\text { Laplacian } \\
\text { Filter }\end{array}$ \\
\hline $\begin{array}{c}\text { Gaussian Filter } \\
(3 \times 3)\end{array}$ & 0.2913 & 0.2384 & 0.4695 & 0.3084 & 0.4616 \\
\hline $\begin{array}{c}\text { Gaussian Filter } \\
(5 \times 5)\end{array}$ & 0.3360 & 0.1897 & 0.4667 & 0.2330 & 0.4487 \\
\hline $\begin{array}{c}\text { Gaussian Filter } \\
(7 \times 7)\end{array}$ & 0.2350 & 0.2176 & 0.4703 & 0.2366 & 0.4368 \\
\hline $\begin{array}{c}\text { Average Filter } \\
(3 \times 3)\end{array}$ & 0.3333 & 0.2890 & 0.4955 & 0.3095 & 0.4731 \\
\hline $\begin{array}{c}\text { Average Filter } \\
(5 \times 5)\end{array}$ & 0.4493 & 0.3019 & 0.4606 & 0.3263 & 0.4556 \\
\hline $\begin{array}{c}\text { Average Filter } \\
(7 \times 7)\end{array}$ & 0.3692 & 0.2524 & 0.4485 & 0.2960 & 0.4586 \\
\hline SNR 5dB & 0.5294 & 0.2769 & 0.4663 & 0.0505 & 0.4263 \\
\hline SNR 10dB & 0.6808 & 0.2318 & 0.5347 & 0.0597 & 0.4437 \\
\hline SNR 20dB & 0.7491 & 0.2304 & 0.6300 & 0.0599 & 0.3785 \\
\hline
\end{tabular}


From Table 3.2, it is observed that, in most of the cases, the Volterra filter produced the highest similarity score among all enhancement algorithms when images are blurred. Laplacian filter also produces comparable performance. When image is corrupted by noise, as shown in Table 3.2, all of the methods reduce similarity score. This fact shows that the image enhancement methods considered in this study also enhance noise. However, among all of methods, Volterra filter still maintains the highest values of similarity score. This observation demonstrates that Volterra filter is more immune from noise compared with other methods.

To generate high similarity score between matching fingerprints is only half of the story. It is also desirable to generate low similarity score between mismatching fingerprints. To get a complete picture, similarity score between reference fingerprint images and mismatching blurred/corrupted fingerprint images and similarity score between reference fingerprint images and mismatching enhanced images are also calculated. It is expected that the best enhancement algorithm would produces the lowest similarity score for each of the cases. For each case, 420 comparisons are made. The resulting mean values of similarity score is shown in Table 3.3. It is observed that, for blurred images, the Volterra filter provides minimum similarity score for mismatching fingerprint images in each of the cases. On the other hand, although Laplacian filter increases similarity score between matching fingerprints as shown in Table 3.2 it also increases similarity score between mismatching fingerprints. This phenomenon indicates that, among four methods considered in this study, the Volterra filter is the best enhancement algorithm for improving the performance of AFM process in case of blurred input fingerprint images. Besides, the Volterra filter does not increase similarity score for mismatching fingerprints corrupted by noise.

Table 3.3

Mean Values of Similarity Score between Reference and Unenhanced (Blurred by Gaussian Filter (size: $3 \times 3,5 \times 5,7 \times 7)$, Average Filter (size: $3 \times 3,5 \times 5,7 \times 7)$ and Corrupted by noise with SNR 5,10 and $20 \mathrm{~dB}$ ) Miss-matched Fingerprint Images and Mean Values of Similarity Score between Reference and Enhanced (using Different Enhancement Algorithms on Blurred/Corrupted Images) Miss-matched Fingerprint Images.

\begin{tabular}{|c|c|c|c|c|c|}
\hline \multirow{3}{*}{$\begin{array}{c}\text { Blurred Input } \\
\text { Fingerprint } \\
\text { Images }\end{array}$} & \multicolumn{5}{|c|}{ Similarity Score } \\
\hline & \multirow{2}{*}{$\begin{array}{c}\text { Without } \\
\text { Enhancement }\end{array}$} & \multicolumn{4}{|c|}{ With Enhancement } \\
\hline & & $\begin{array}{l}\text { Gabor } \\
\text { Filter }\end{array}$ & $\begin{array}{c}\text { Volterra } \\
\text { Filter }\end{array}$ & $\begin{array}{l}\text { Combination } \\
\text { Filter }\end{array}$ & $\begin{array}{l}\text { Laplacian } \\
\text { Filter }\end{array}$ \\
\hline $\begin{array}{c}\text { Gaussian Filter } \\
(3 \times 3)\end{array}$ & 0.1281 & 0.1535 & 0.0163 & 0.1789 & 0.2774 \\
\hline $\begin{array}{c}\text { Gaussian Filter } \\
(5 \times 5)\end{array}$ & 0.1495 & 0.1283 & 0.0167 & 0.1394 & 0.2750 \\
\hline $\begin{array}{c}\text { Gaussian Filter } \\
(7 \times 7)\end{array}$ & 0.1230 & 0.1300 & 0.0163 & 0.1453 & 0.2716 \\
\hline $\begin{array}{c}\text { Average Filter } \\
(3 \times 3)\end{array}$ & 0.2328 & 0.1982 & 0.1873 & 0.1863 & 0.2809 \\
\hline
\end{tabular}




\begin{tabular}{|c|c|c|c|c|c|}
\hline $\begin{array}{c}\text { Average Filter } \\
\text { (5× 5) }\end{array}$ & 0.2156 & 0.1906 & 0.0372 & 0.2222 & 0.2812 \\
\hline $\begin{array}{c}\text { Average Filter } \\
(7 \times 7)\end{array}$ & 0.1966 & 0.1573 & 0.0182 & 0.1878 & 0.2739 \\
\hline SNR 5db & 0.2965 & 0.1893 & 0.2968 & 0.0366 & 0.2886 \\
\hline SNR 10db & 0.2793 & 0.1711 & 0.2792 & 0.0355 & 0.2746 \\
\hline SNR 20db & 0.2657 & 0.1638 & 0.2659 & 0.0324 & 0.2118 \\
\hline
\end{tabular}

The False Nonmatching Rate (FNMR) can be defined as the probability of failing to identify a matching fingerprint and the False Matching Rate (FMR) can be defined as the probability of claiming a non-matching fingerprint as a matching fingerprint. A good AFM system needs to guarantee low FNMR and FMR [26]. We use the mean and standard deviation of similarity score between matching and mismatching fingerprints to estimate FNMR and FMR assuming the distribution of similarity score is a Gaussian distribution. To conduct this estimation, the similarity score between reference and matching blurred/corrupted fingerprint images are taken as one data set while similarity score between reference and mismatching blurred/corrupted input fingerprint images are taken as another data set. Based on histograms of these two data set, two probability density functions (pdf) are plotted on the same figure. Two pdf curves intersect at a point whose value is used as the threshold. The mean $(\mu)$, standard deviation $(\sigma)$ and threshold point $(\delta)$ are considered while calculating the probability of error, $P_{E}$ for both evaluation approaches.

$P_{E}=\left\{\begin{array}{l}0.5-\operatorname{erf}\left(\frac{\mu-\delta}{\sigma}\right) ; \text { for } \delta \leq \mu \\ 0.5+\operatorname{erf}\left(\frac{\delta-\mu}{\sigma}\right) ; \text { for } \delta \geq \mu\end{array}\right.$

Though FNMR and FMR both are computed using the formulae of $P_{E}$, the value of $\mu$ and $\sigma$ are different for each cases. In case of FNMR, the mean and standard deviation of similarity score between matching fingerprint images are considered as $\mu$ and $\sigma$ respectively in the equations of $P_{E}$ while in case of computing FMR the mean and standard deviation of mismatching fingerprint images are considered as $\mu$ and $\sigma$ respectively in the equations of $P_{E}$. The intersection point of two pdf curves is used as the threshold for both cases. 


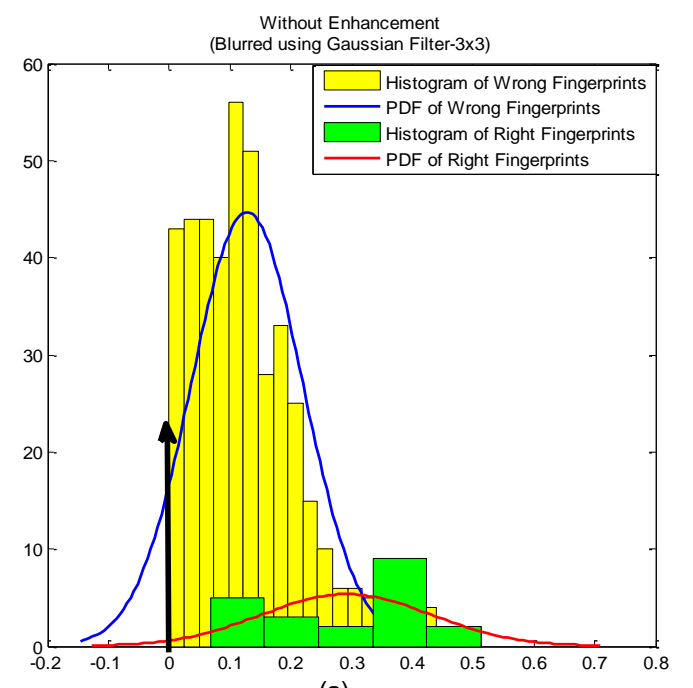

(a)

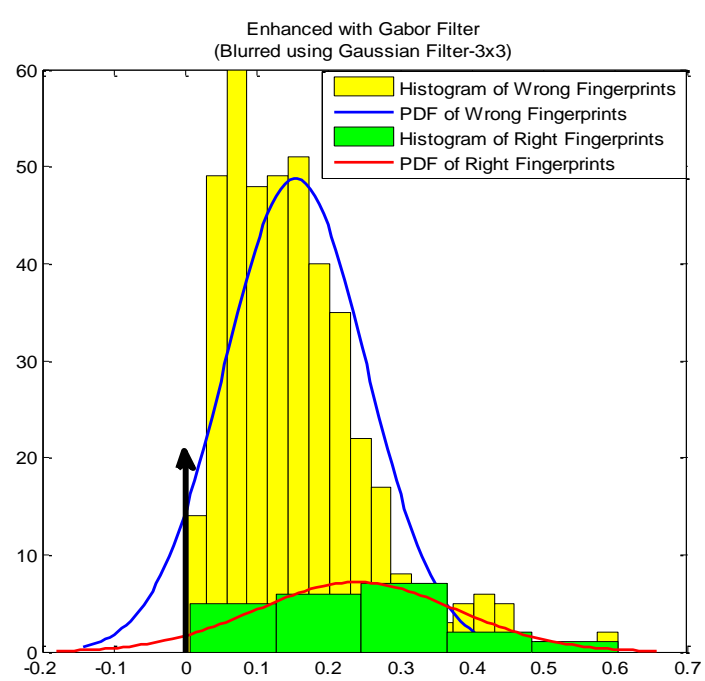

(b)

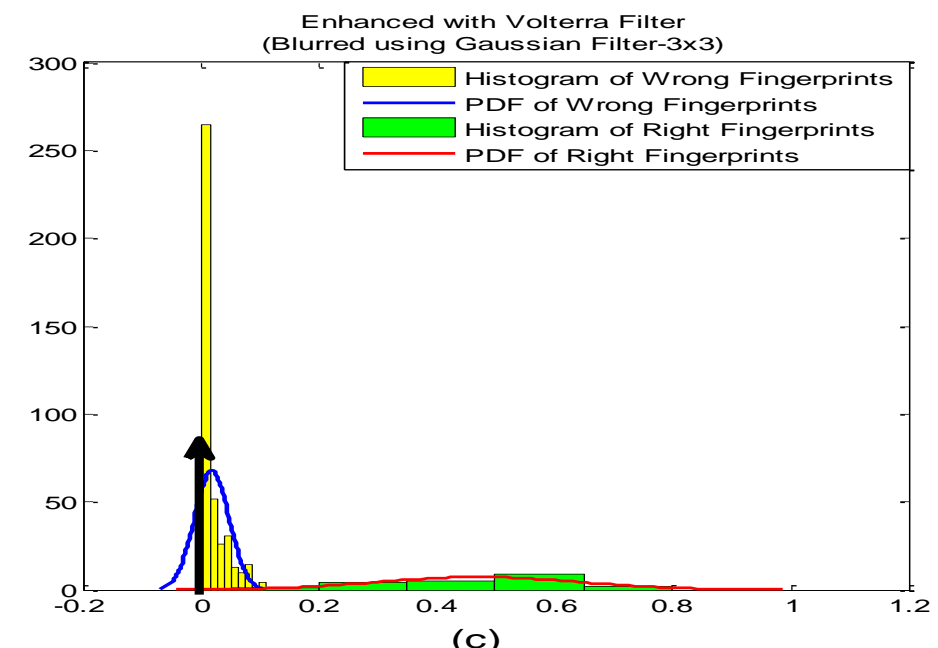

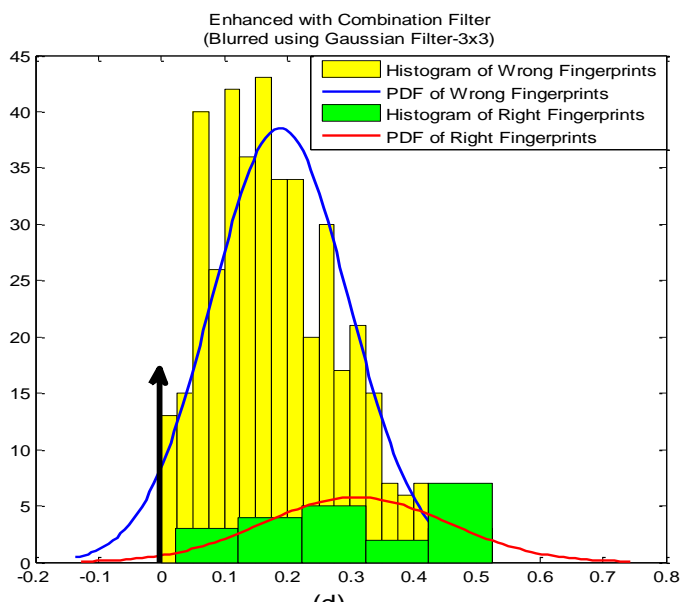

(d)

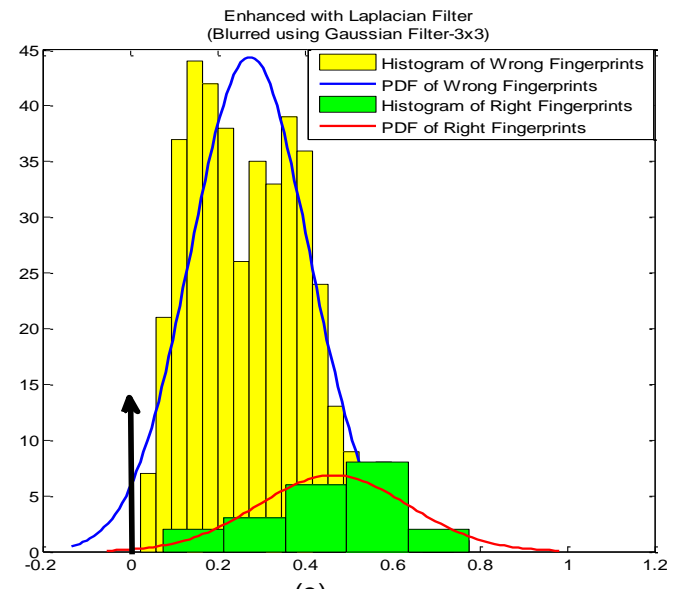

(e)

Figure 3.4: Histogram and Probability Density Function (PDF) representation for with and without fingerprint image enhancement algorithms in case of blurred (using Gaussian Filter- $3 \times$ 3 ) input fingerprint images. 


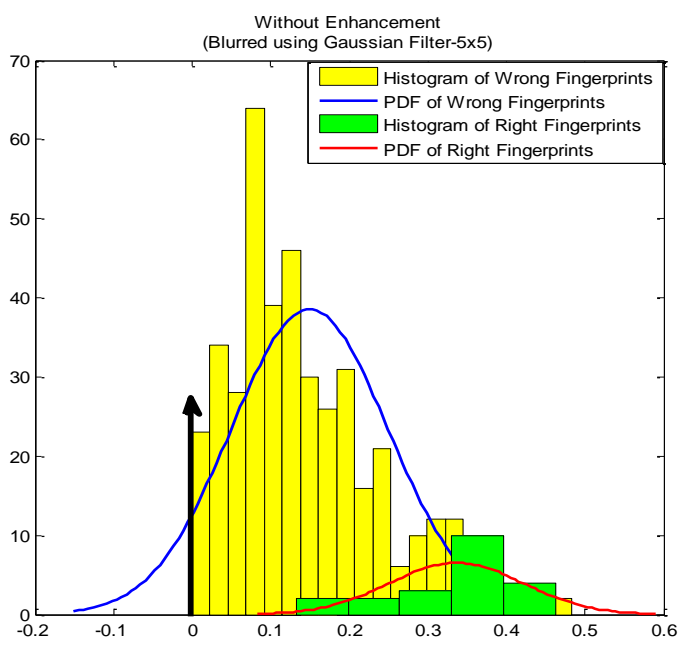

(a)

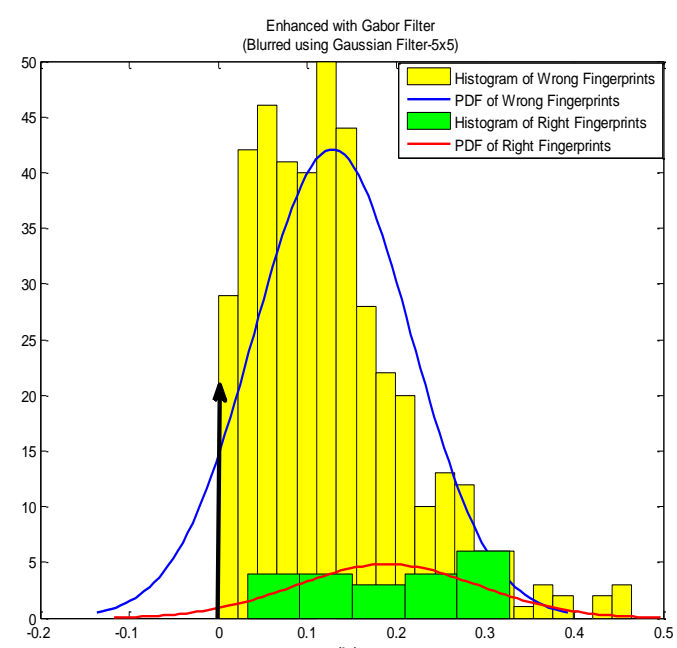

(b)

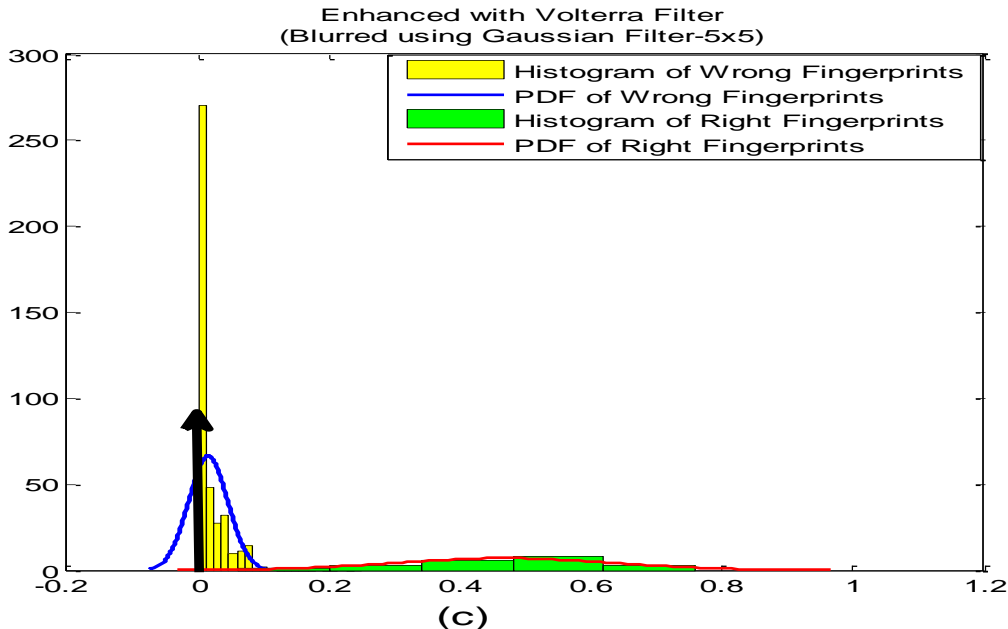

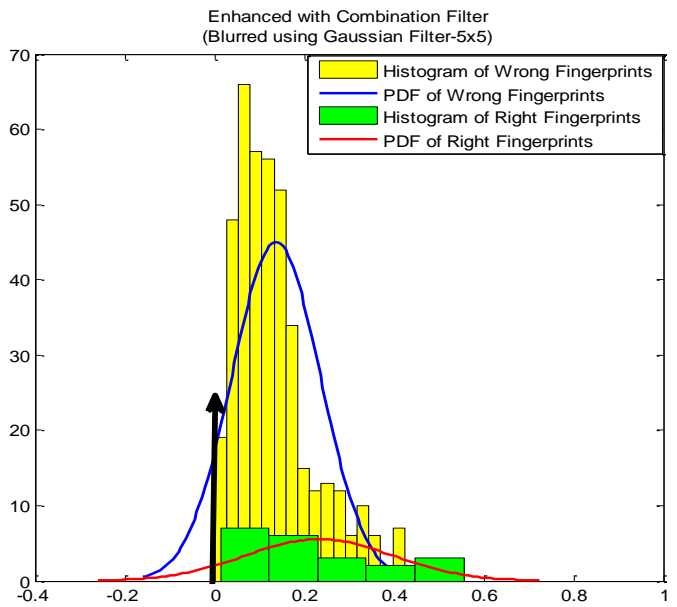

(d)

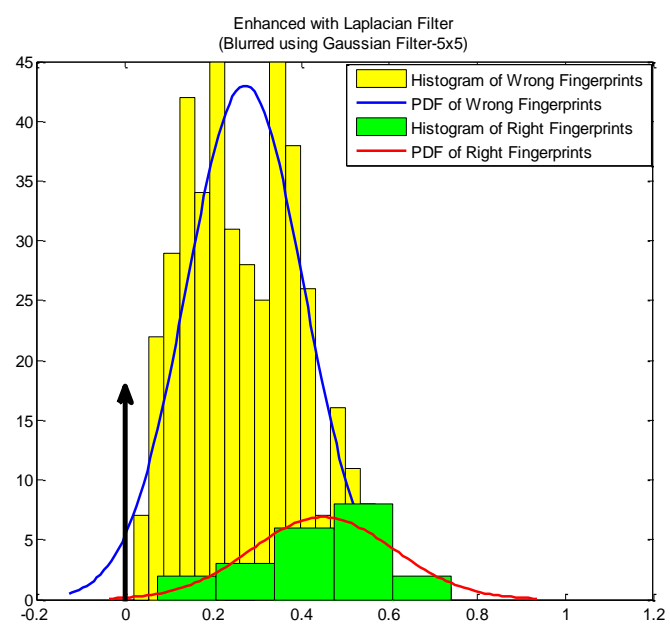

(e)

Figure 3.5: Histogram and Probability Density Function (PDF) representation for with and without fingerprint image enhancement algorithms in case of blurred (using Gaussian Filter- $5 \times$ 5 ) input fingerprint images. 


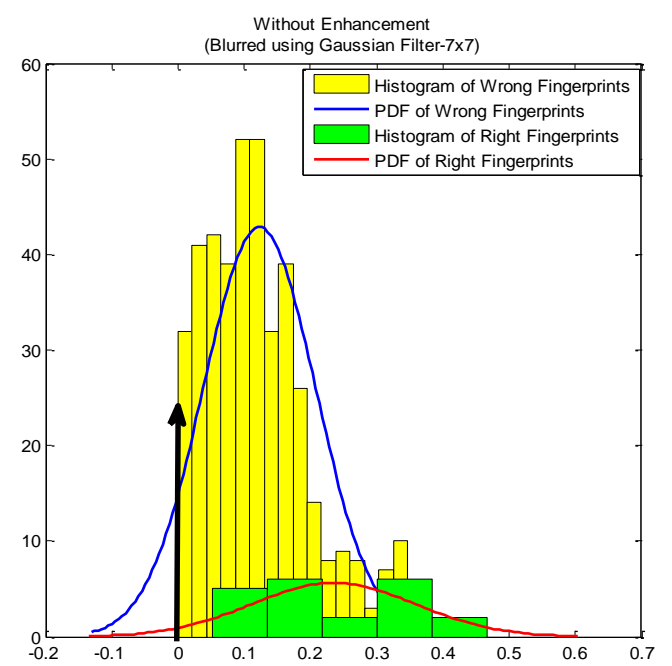

(a)

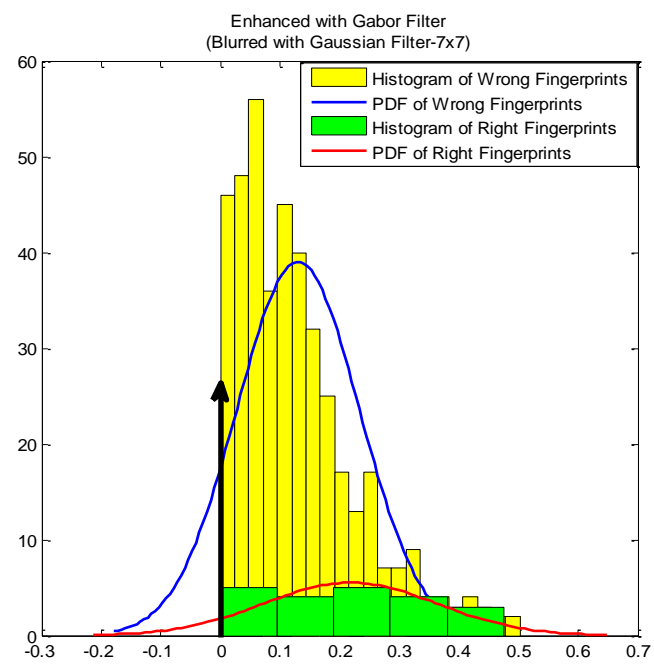

(b)

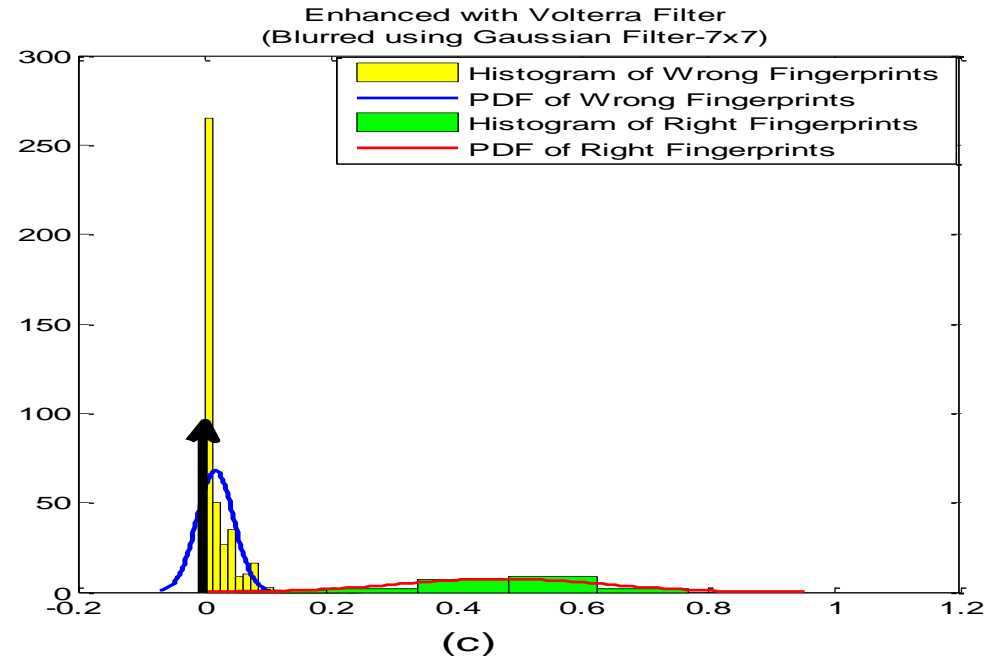

Enhanced with Combination Filter

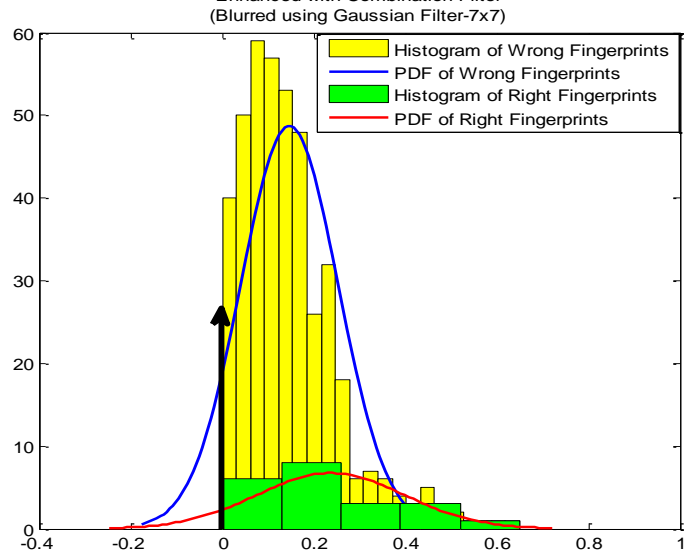

(d)

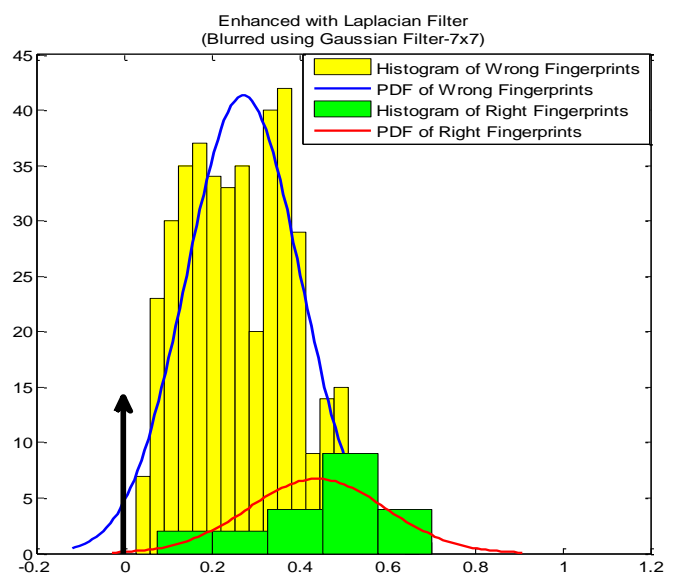

(e)

Figure 3.6: Histogram and Probability Density Function (PDF) representation for with and without fingerprint image enhancement algorithms in case of blurred (using Gaussian Filter- $7 \times$ 7) input fingerprint images. 

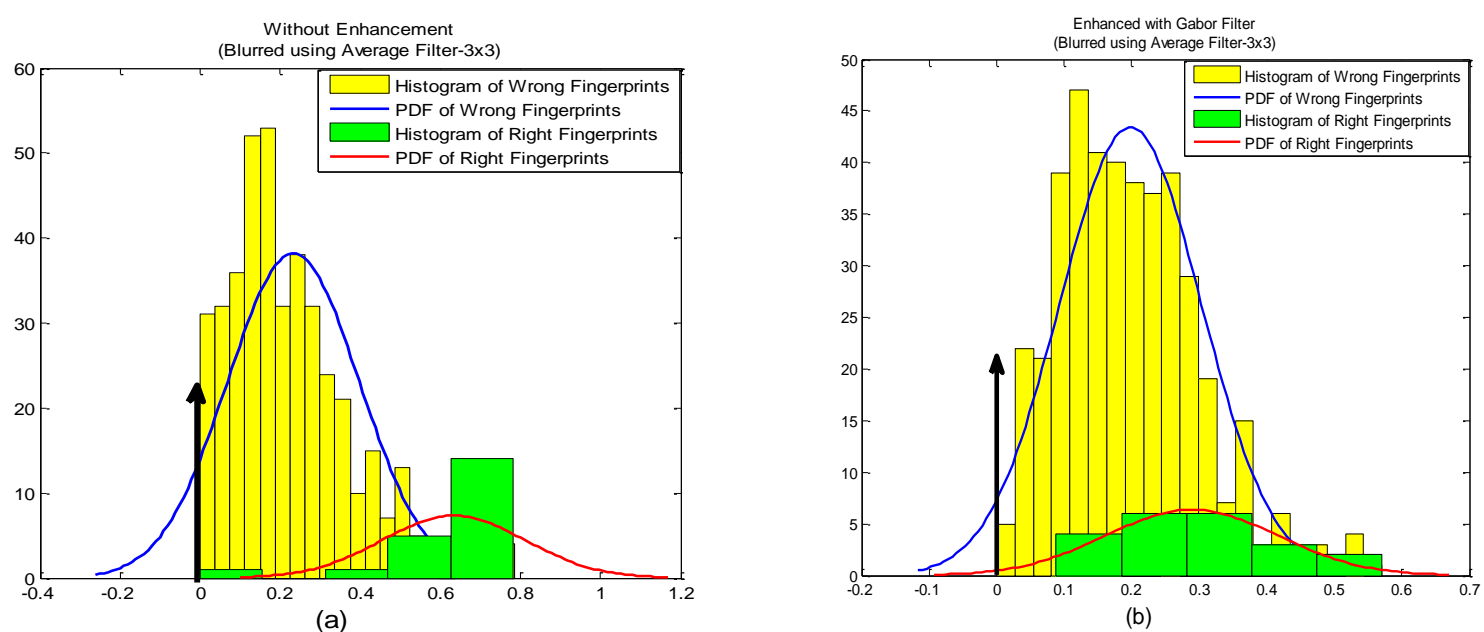

(a)

Enhanced with Volterra Filter

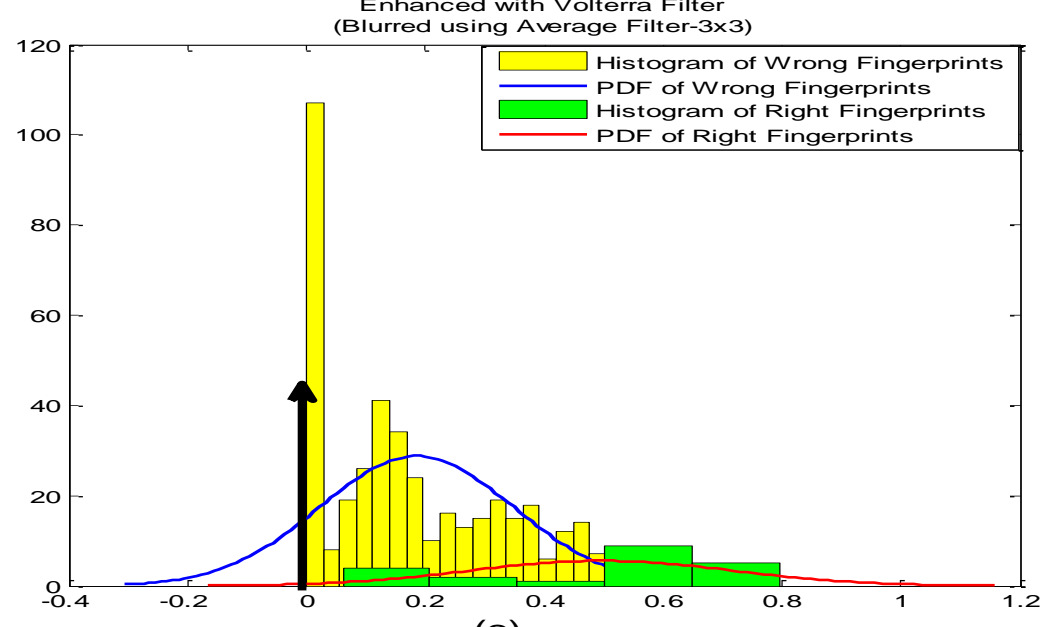

(c)
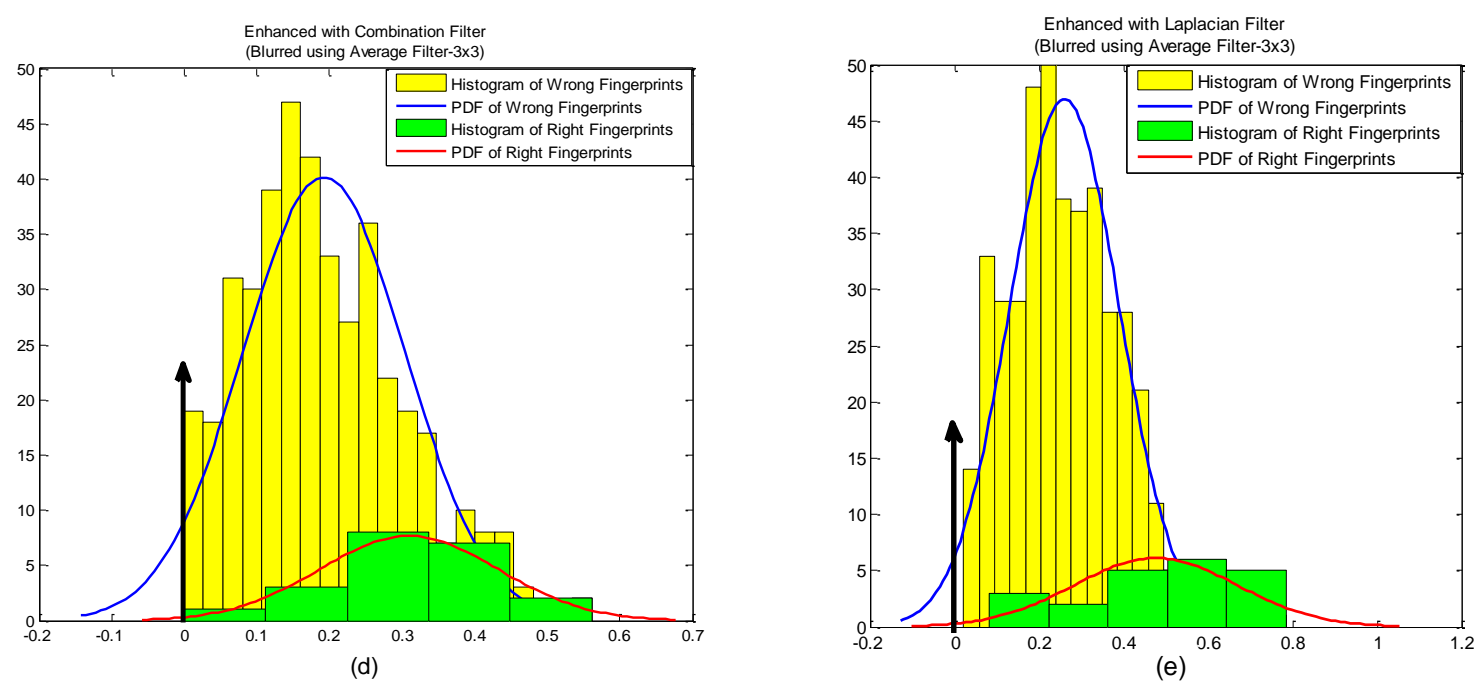

Figure 3.7: Histogram and Probability Density Function (PDF) representation for with and without fingerprint image enhancement algorithms in case of blurred (using Average Filter- $3 \times$ 3 ) input fingerprint images. 


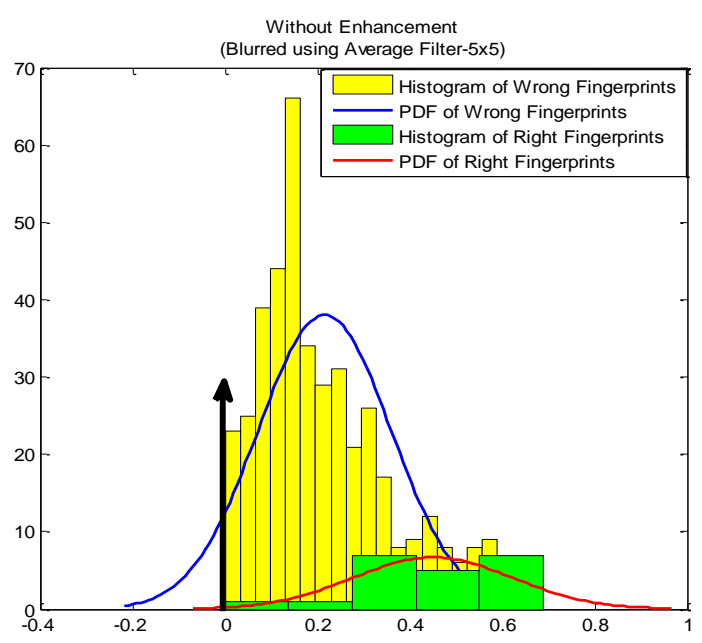

(a)

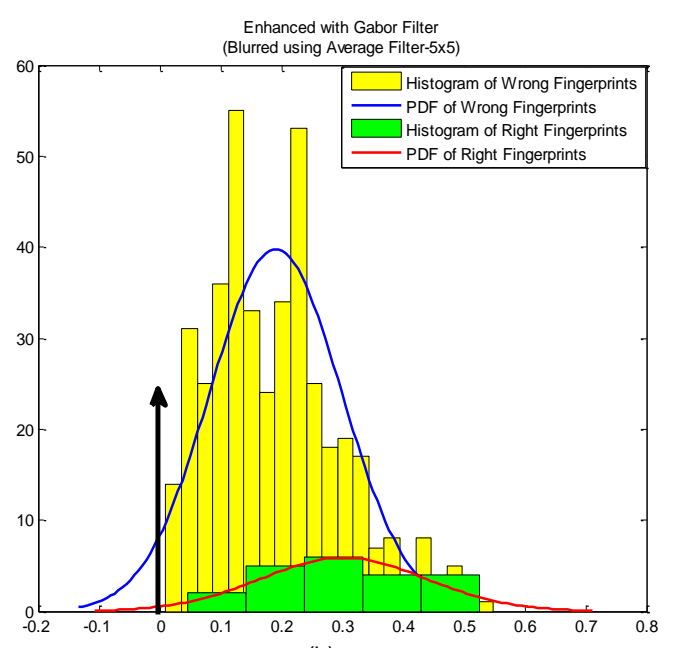

(b)

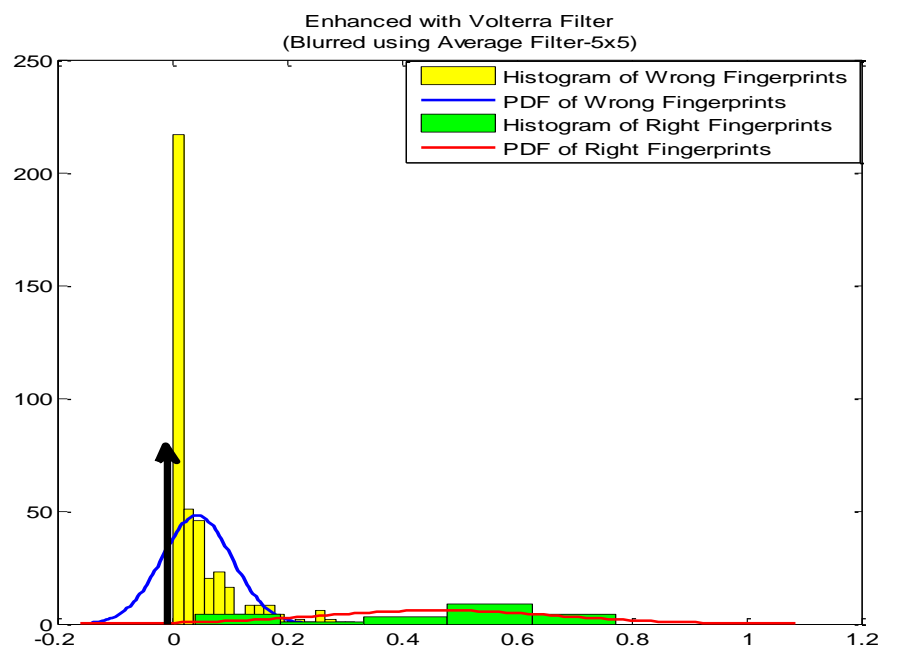

(c)
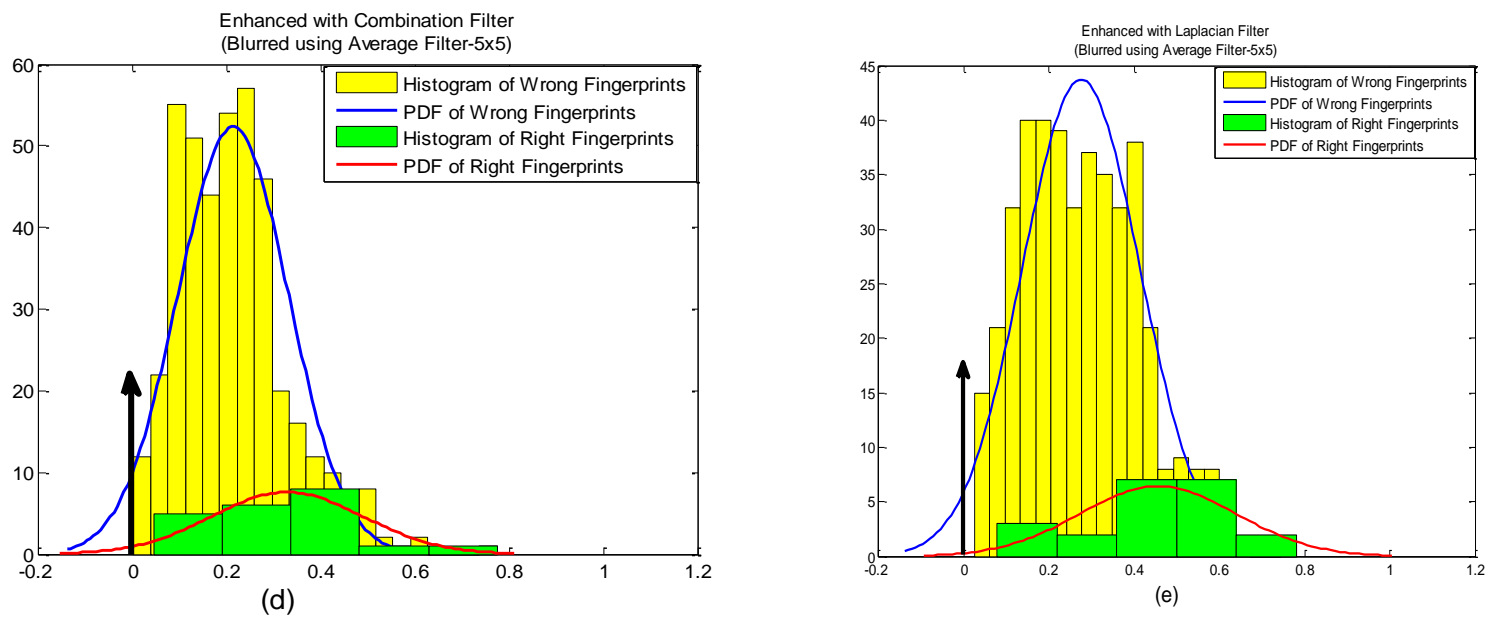

(e)

Figure 3.8: Histogram and Probability Density Function (PDF) representation for with and without fingerprint image enhancement algorithms in case of blurred (using Average Filter- $5 \times$ 5 ) input fingerprint images. 


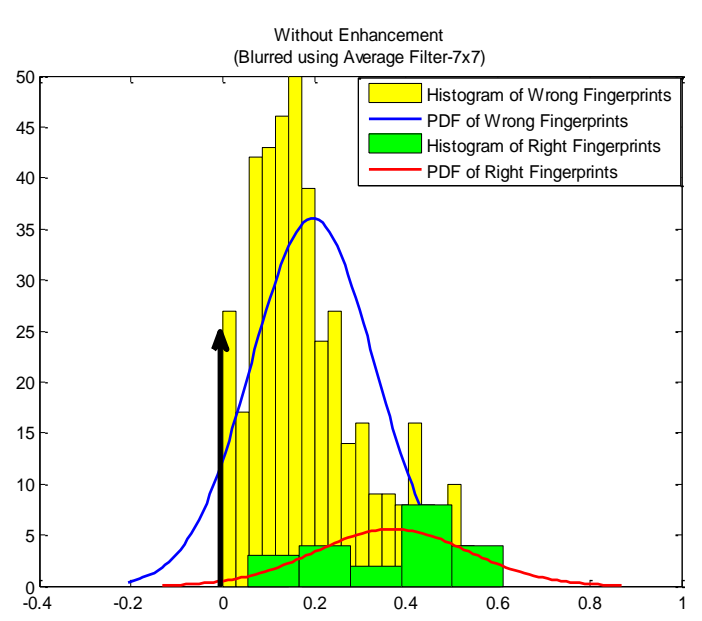

(a)

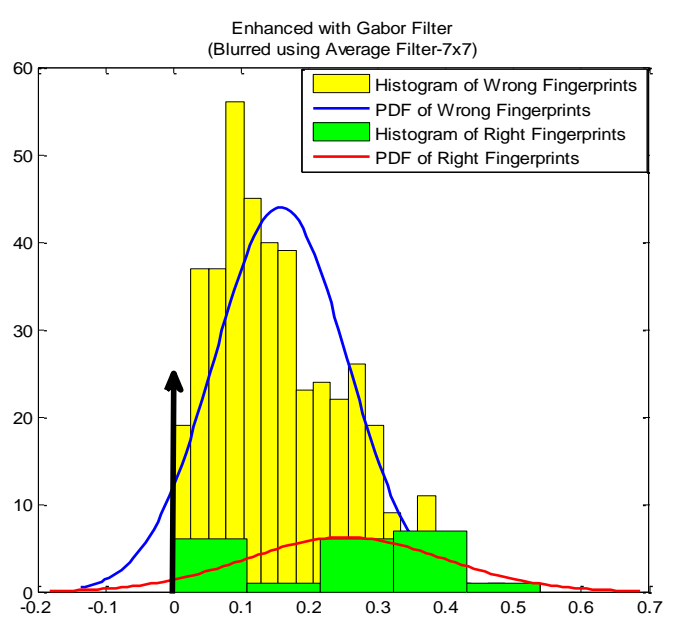

(b)

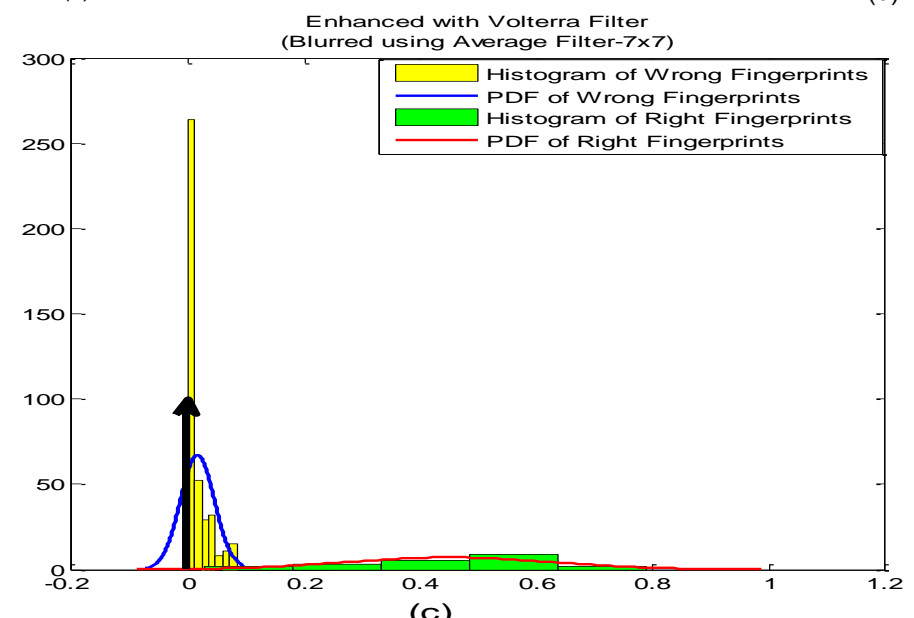

(c)
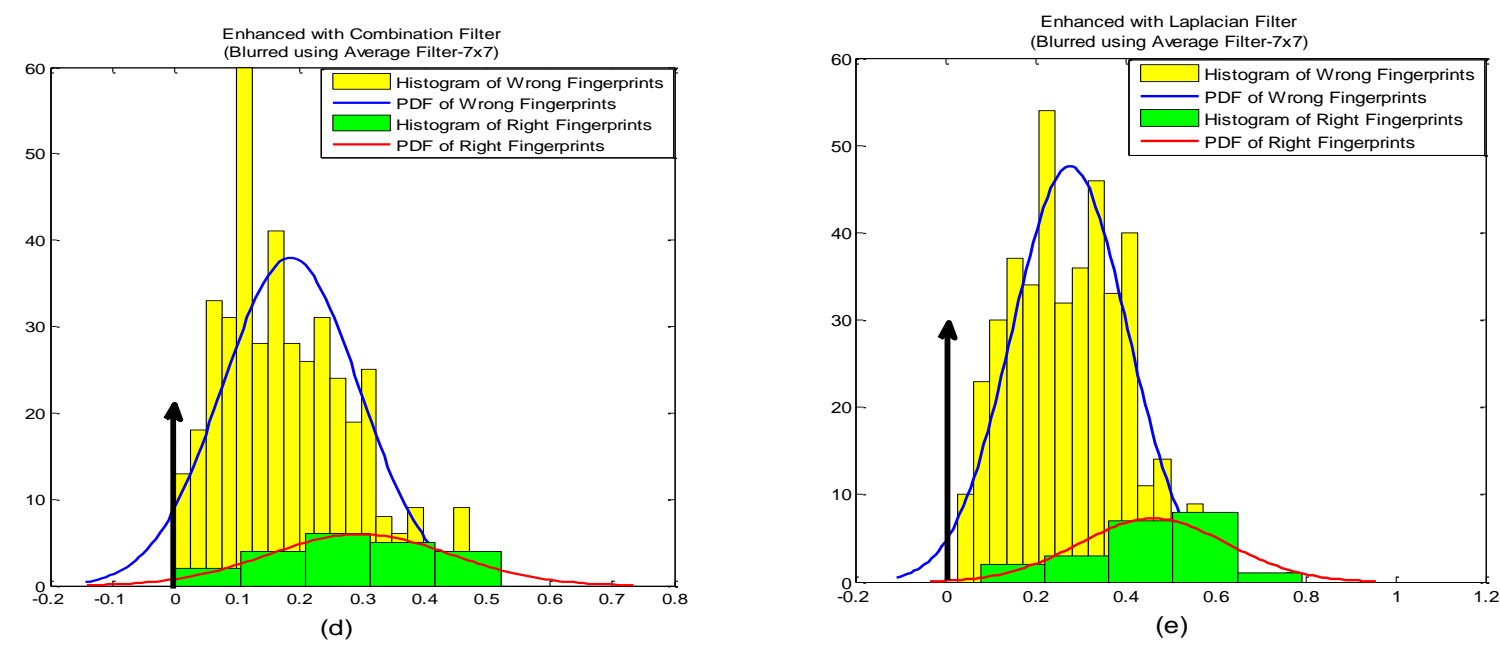

Figure 3.9: Histogram and Probability Density Function (PDF) representation for with and without fingerprint image enhancement algorithms in case of blurred (using Average Filter- $7 \times$ 7) input fingerprint images. 

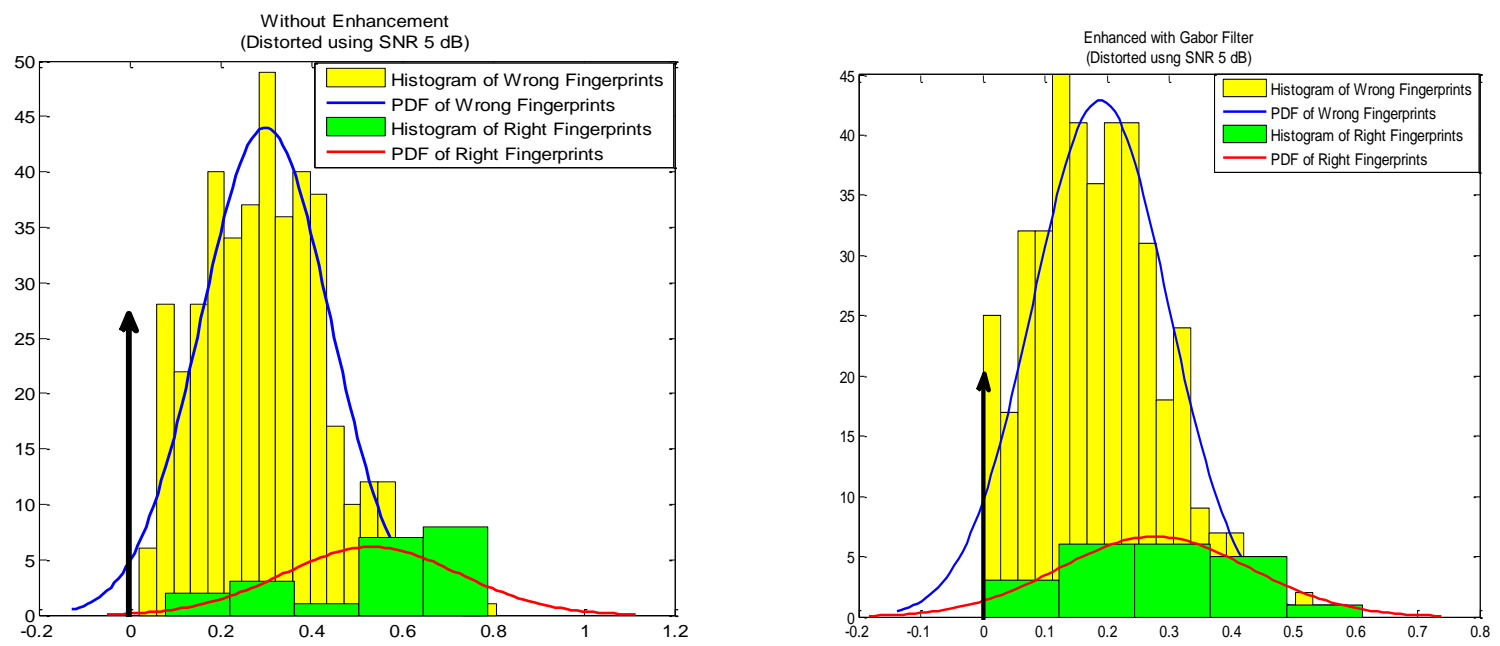

(a)

(b)

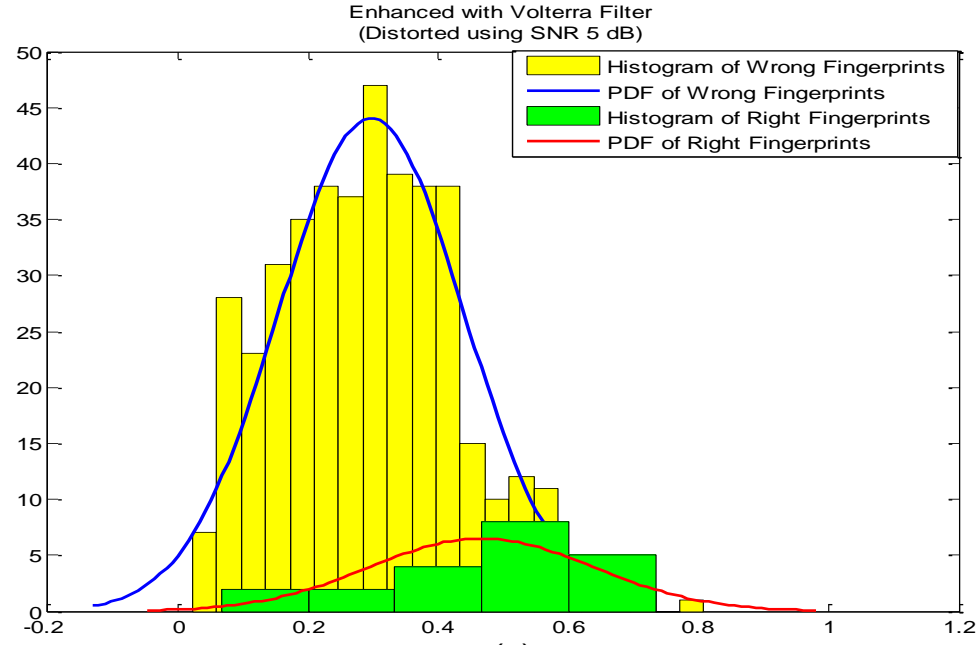

(c)

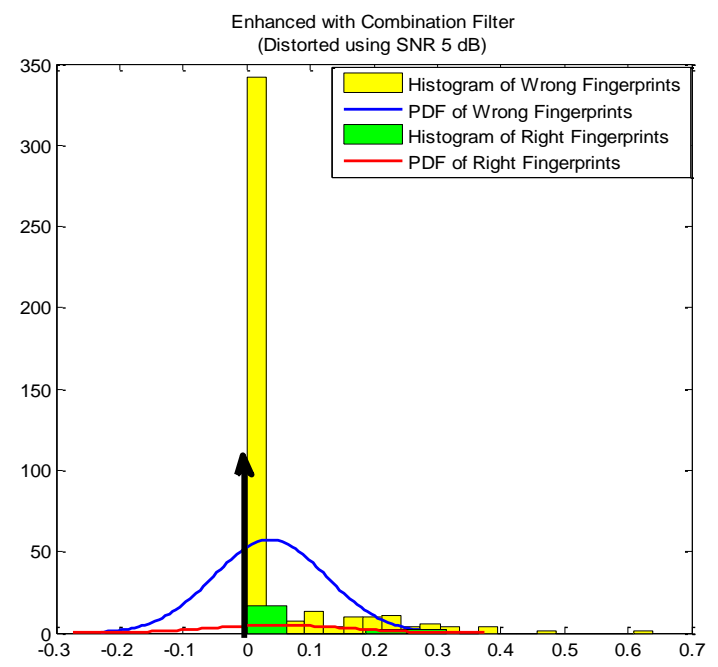

(d)

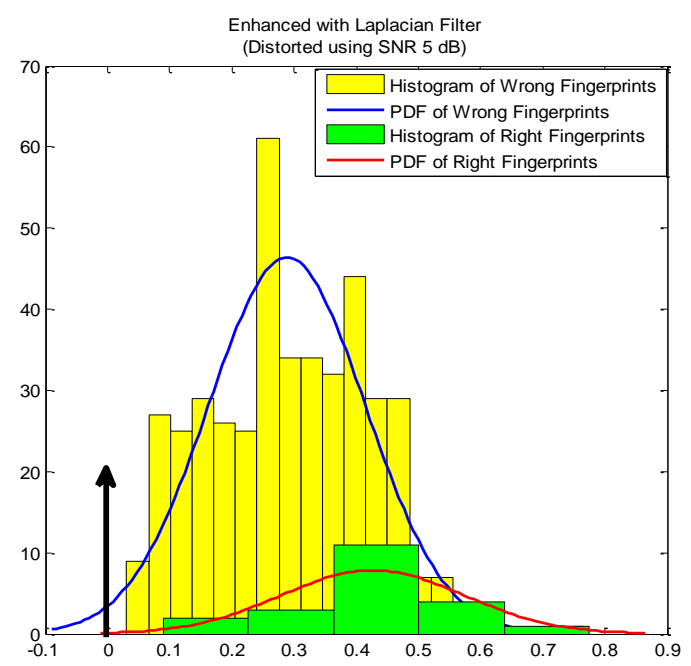

(e)

Figure 3.10: Histogram and Probability Density Function (PDF) representation for with and without fingerprint image enhancement algorithms in case of noisy (SNR $5 \mathrm{~dB}$ ) input fingerprint images. 

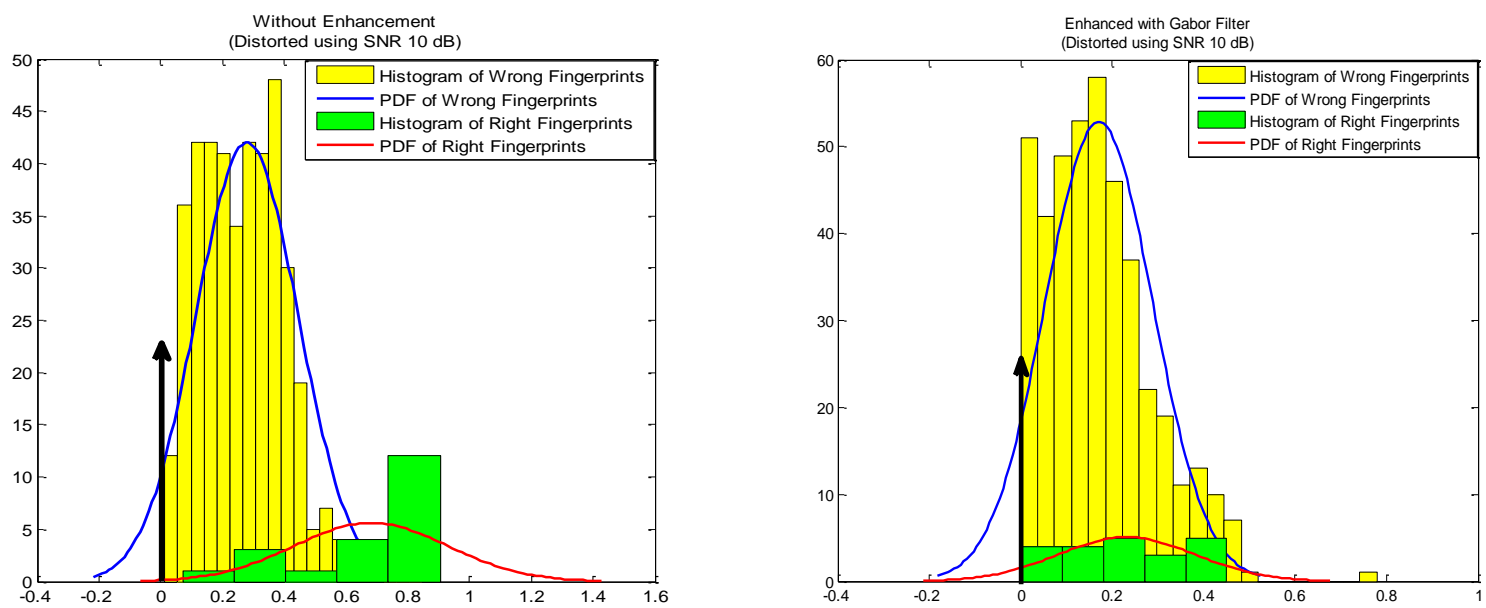

(a)

Enhanced with Volterra Filter

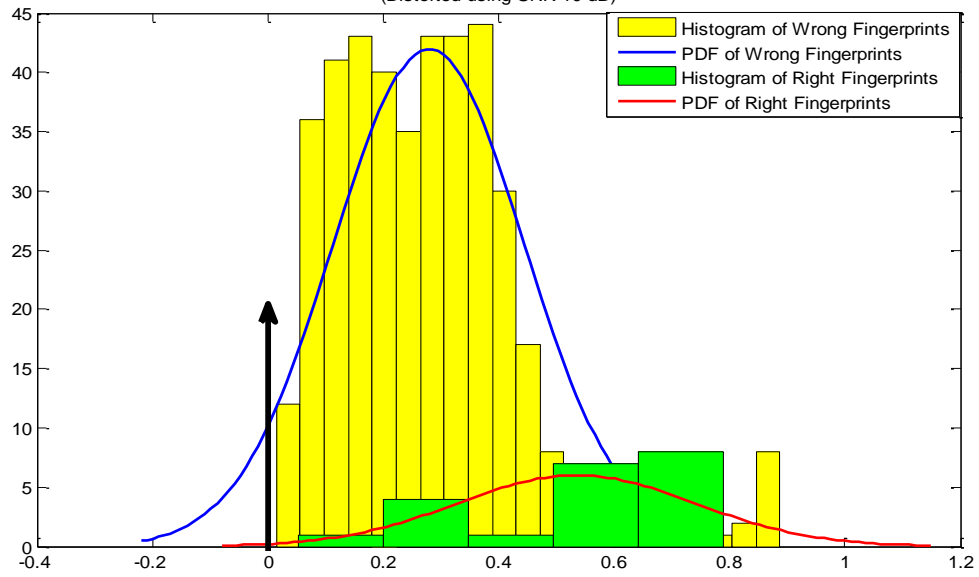

(c)

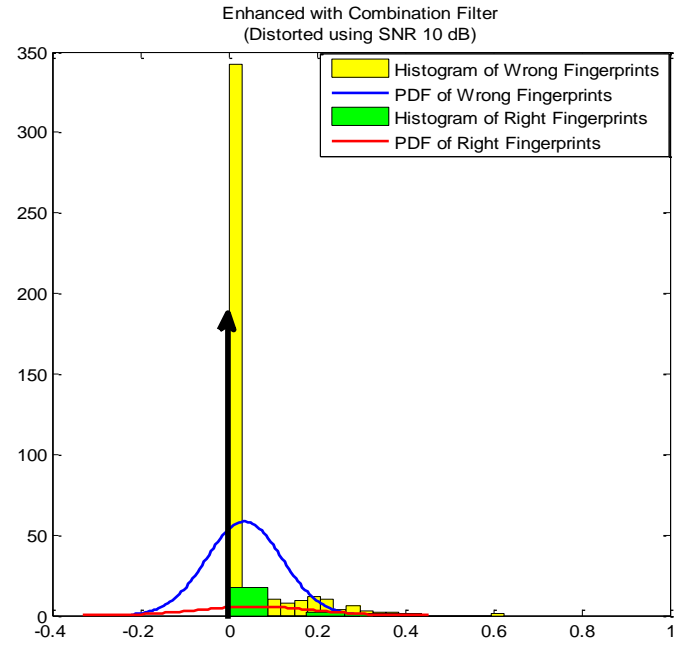

(d)

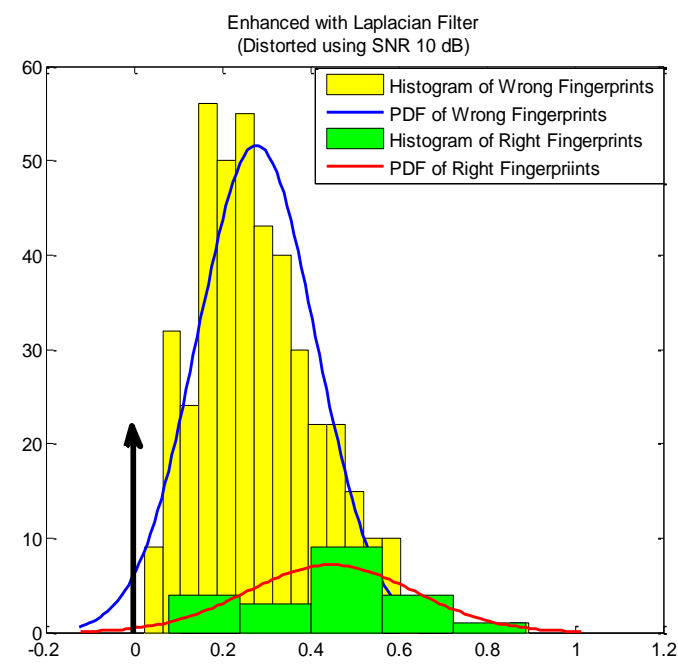

(e)

Figure 3.11: Histogram and Probability Density Function (PDF) representation for with and without fingerprint image enhancement algorithms in case of noisy (SNR $10 \mathrm{~dB}$ ) input fingerprint images. 

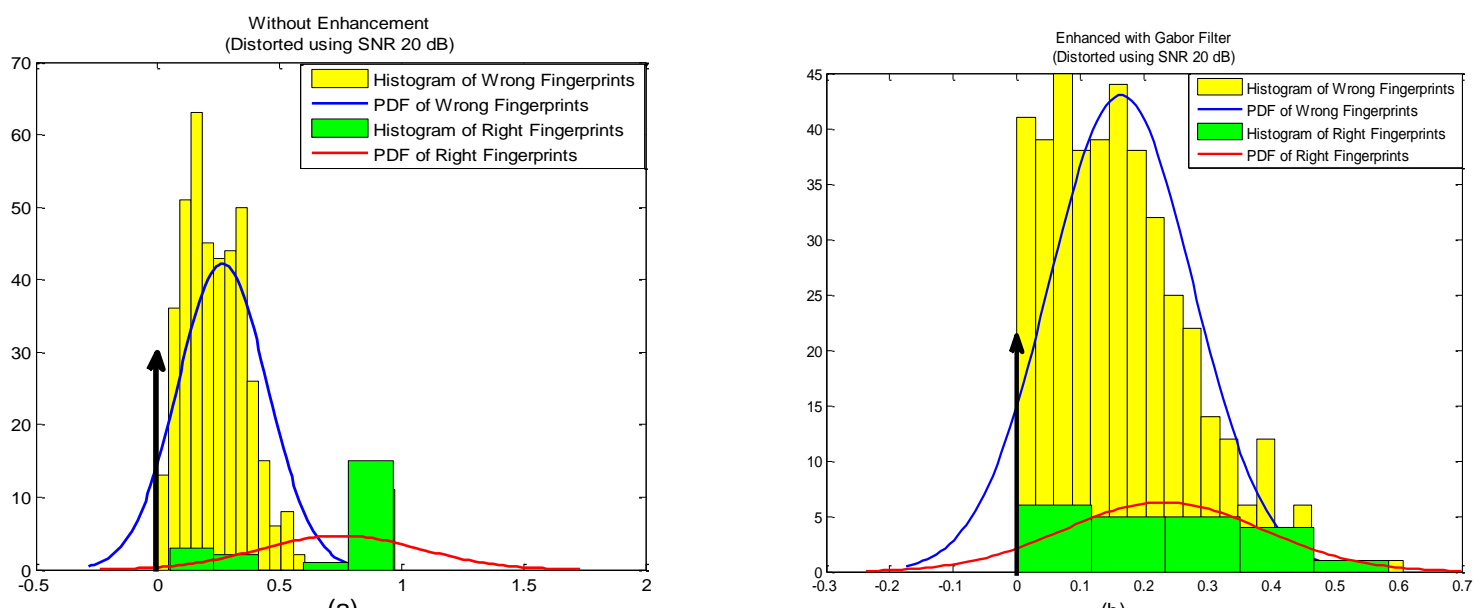

(a)

(b)

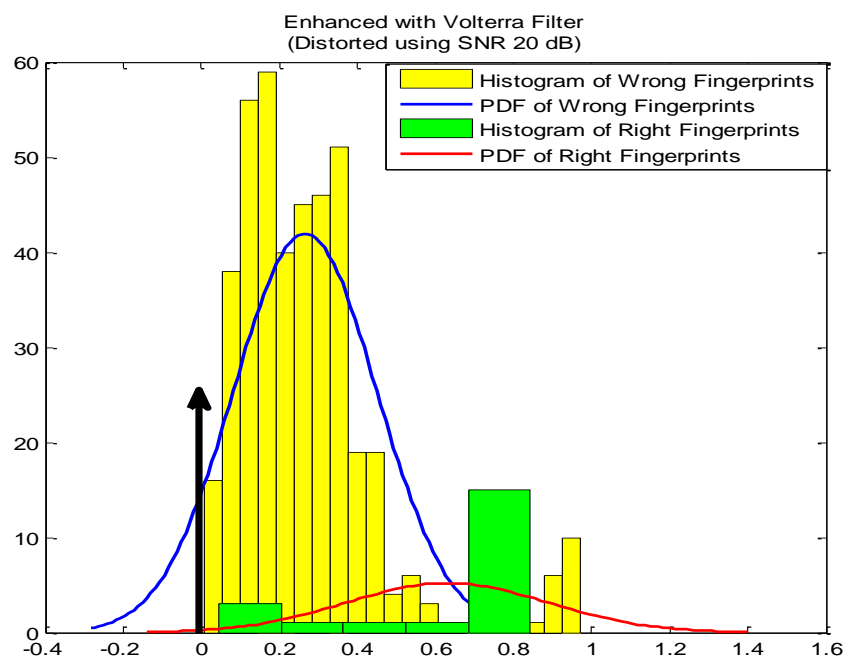

(c)
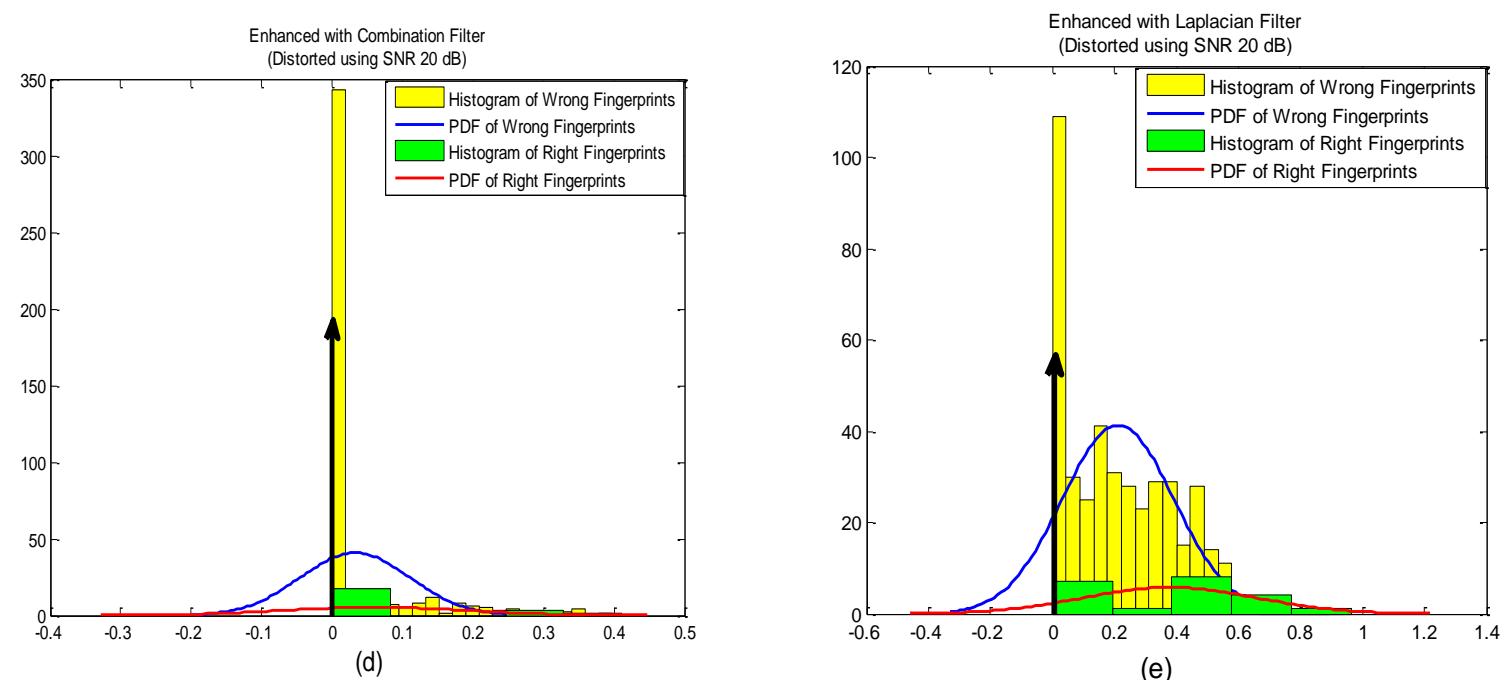

(e)

Figure 3.12: Histogram and Probability Density Function (PDF) representation for with and without fingerprint image enhancement algorithms in case of noisy (SNR $20 \mathrm{~dB}$ ) input fingerprint images. 
Figures 3.4-3.12 represents the histogram as well as probability density function (pdf) of similarity score for different fingerprint enhancement algorithms in case of different blurred/corrupted input fingerprint images. The intersection point of both curves is considered as the threshold point. FNMR is estimated with the pdf of similarity scores of 21 matching fingerprint comparisons and FMR is estimated with the pdf of similarity scores of 420 non-matching fingerprint comparisons. The calculation of FNMR and FMR is conducted for different fingerprint enhancement algorithms in each scenario and the results are listed in Table 3.4. From Table 3.4, it is clear that the Volterra filter decreases FNMR and FMR for each case of blurred images and achieve the lowest FNR and FMR among all of image enhancement algorithms. For fingerprint corrupted by noise, the Volterra filer reduces FMR but increase FNMR. However, among all of image enhancement algorithms under investigation, the Volterra filter still outperforms all of other methods. This finding can be explained as following. To enhancement details of an image, the noise might be amplified at the same time. However, among all of image enhancement considered in this article, the Volterra filer is most immune to the noise and it delivers the best performance when processing blurred images.

Table 3.4

Comparison of Probability of Error (FNMR and FMR)

\begin{tabular}{|c|c|c|c|}
\hline Type of Distortion & $\begin{array}{c}\text { Name of Distortion and } \\
\text { Enhancement } \\
\text { Algorithms }\end{array}$ & $\begin{array}{l}\text { False Nonmatching } \\
\text { Rate (FNMR) }\end{array}$ & $\begin{array}{c}\text { False } \\
\text { Matching } \\
\text { Rate (FMR) }\end{array}$ \\
\hline \multirow{5}{*}{$\begin{array}{l}\text { Distorted using } \\
\text { Gaussian Filter } \\
\quad(3 \times 3)\end{array}$} & Distorted & 0.54 & 0.028 \\
\hline & Gabor Filter & 0.66 & 0.066 \\
\hline & Volterra Filter & 0.02 & 0.001 \\
\hline & Volterra + Gabor Filter & 0.62 & 0.054 \\
\hline & Laplacian Filter & 0.64 & 0.035 \\
\hline \multirow{5}{*}{$\begin{array}{l}\text { Distorted using } \\
\text { Gaussian Filter } \\
\qquad(5 \times 5)\end{array}$} & Distorted & 0.42 & 0.044 \\
\hline & Gabor Filter & 0.24 & 0.006 \\
\hline & Volterra Filter & 0.01 & 0.000 \\
\hline & Volterra + Gabor Filter & 0.76 & 0.002 \\
\hline & Laplacian Filter & 0.67 & 0.032 \\
\hline \multirow{5}{*}{$\begin{array}{l}\text { Distorted using } \\
\text { Gaussian Filter } \\
(7 \times 7)\end{array}$} & Distorted & 0.69 & 0.023 \\
\hline & Gabor Filter & 0.82 & 0.016 \\
\hline & Volterra Filter & 0.01 & 0.002 \\
\hline & Volterra + Gabor Filter & 0.82 & 0.013 \\
\hline & Laplacian Filter & 0.71 & 0.028 \\
\hline \multirow{5}{*}{$\begin{array}{l}\text { Distorted using } \\
\text { Average Filter } \\
\qquad(3 \times 3)\end{array}$} & Distorted & 0.83 & 0.049 \\
\hline & Gabor Filter & 0.90 & 0.060 \\
\hline & Volterra Filter & 0.46 & 0.035 \\
\hline & Volterra + Gabor Filter & 0.92 & 0.045 \\
\hline & Laplacian Filter & 0.56 & 0.044 \\
\hline \multirow{4}{*}{$\begin{array}{l}\text { Distorted using } \\
\text { Average Filter } \\
(5 \times 5)\end{array}$} & Distorted & 0.58 & 0.032 \\
\hline & Gabor Filter & 0.82 & 0.016 \\
\hline & Volterra Filter & 0.10 & 0.004 \\
\hline & Volterra + Gabor Filter & 0.83 & 0.014 \\
\hline
\end{tabular}




\begin{tabular}{|c|c|c|c|}
\hline & Laplacian Filter & 0.69 & 0.026 \\
\hline \multirow{5}{*}{$\begin{array}{l}\text { Distorted using } \\
\text { Average Filter } \\
\quad(7 \times 7)\end{array}$} & Distorted & 0.74 & 0.018 \\
\hline & Gabor Filter & 0.82 & 0.011 \\
\hline & Volterra Filter & 0.03 & 0.002 \\
\hline & Volterra + Gabor Filter & 0.80 & 0.018 \\
\hline & Laplacian Filter & 0.92 & 0.023 \\
\hline \multirow{5}{*}{$\begin{array}{c}\text { Corrupted by } \\
\text { Noise }(\text { SNR } 5 \mathrm{~dB})\end{array}$} & Distorted & 0.60 & 0.022 \\
\hline & Gabor Filter & 0.83 & 0.018 \\
\hline & Volterra Filter & 0.79 & 0.015 \\
\hline & Volterra + Gabor Filter & 0.96 & 0.032 \\
\hline & Laplacian Filter & 0.80 & 0.023 \\
\hline \multirow{5}{*}{$\begin{array}{c}\text { Corrupted by } \\
\text { Noise (SNR } 10 \\
\text { dB) }\end{array}$} & Distorted & 0.38 & 0.026 \\
\hline & Gabor Filter & 0.96 & 0.009 \\
\hline & Volterra Filter & 0.65 & 0.005 \\
\hline & Volterra + Gabor Filter & 0.84 & 0.026 \\
\hline & Laplacian Filter & 0.76 & 0.012 \\
\hline \multirow{5}{*}{$\begin{array}{c}\text { Corrupted by } \\
\text { Noise (SNR } 20 \\
\text { dB) }\end{array}$} & Distorted & 0.34 & 0.025 \\
\hline & Gabor Filter & 0.85 & 0.018 \\
\hline & Volterra Filter & 0.48 & 0.015 \\
\hline & Volterra + Gabor Filter & 0.85 & 0.028 \\
\hline & Laplacian Filter & 0.79 & 0.032 \\
\hline
\end{tabular}




\section{Chapter 4}

\section{Conclusion}

To improve the accuracy of fingerprint identification, fingerprint enhancement is crucial. One of the common issues of fingerprint identification system is that fingerprint image might be blurred due to different reasons. In this study, four different image enhancement methods: Gabor filter, Laplacian filter, Volterra filter and the combination of Volterra and Gabor filter are investigated. Twenty-one good quality fingerprints from FVC 2004 database are used is this study. The good quality images are blurred by either Gaussian or average filters with different sizes or corrupted by noise with different SNR settings. Different image enhancement algorithms are then applied to process blurred/corrupted fingerprints. The similarity scores between reference fingerprints and matching or mismatching fingerprints are calculated. Our results show that among all of the image enhancement algorithms considered in this study, Volterra filter delivers the best performance in terms of reducing both FNMR and FMR for blurred images. When image is corrupted by noise rather than blurred, the image enhancement algorithm does not necessarily improve the system performance. However, the Volterra filter still shows the greatest immunity to noise among all of the image enhancement algorithms considered in this study. It can be concluded that the Volterra filter is a good fingerprint enhancement algorithm with good potential and deserve further study.

As Volterra filter is proved to be a potential fingerprint image enhancement algorithm, possible future study might include the implementation of higher-order Volterra filter as a fingerprint image enhancement algorithm in case of blurred input fingerprint images and observe the performance of AFM process using a higher-order Volterra filter. 


\section{Appendix A- Evaluation method using Quality Factor (QF)}

One possible evaluation method of fingerprint enhancement algorithm can be defined as computation of QF for each enhancement algorithms and compare them with each other. If $I(i, j)$ is a gray scale image of input fingerprint image, then the Fourier transform of image $I(i, j)$ is defined as

$I(u, v)=\frac{1}{M N} \sum_{i=0}^{M-1} \sum_{j=0}^{N-1} I(i, j) e^{-i 2 \pi\left(\frac{u i}{M}+\frac{v j}{N}\right)}$

where, $M$ and $N$ is the size of image $I(i, j)$. The power spectrum of image $I(i, j)$ is defined as

$P(u, v)=|I(u, v)|^{2}$

QF of image $I(i, j)$ is going to be computed from the power spectrum $(P(u, v))[22] . P(u, v)$ is basically a circular representation of ridge frequency ranging from 0.06 to 0.5 [17]. A good quality fingerprint image represents a strong ring pattern in $P(u, v)$ whereas a poor quality fingerprint is going to represent a weak ring pattern in $P(u, v)$. This ring pattern is separated using a Butterworth low pass filter. A Butterworth function is defined as

$H(u, v \mid m, n)=\frac{1}{1+\frac{1}{m^{2 n}}\left(\left(\frac{u-a}{M}\right)^{2}+\left(\frac{v-b}{N}\right)^{2}\right)^{n}}$

where, $m$ and $n$ are the indices of Butterworth function, $(u, v)$ is the pixel index of power spectrum in the frequency domain and $(a, b)$ is the location of the center of the power spectrum. By taking differences between two Butterworth low pass filters, a total of $T$ equally spaced bandpass filters, $B P_{E}$ are achieved [9].

$B P_{E}(u, v)=H\left(u, v \mid m_{t+1}, n\right)-H\left(u, v \mid m_{t}, n\right)$

where, $m_{t}=0.06+t 0.5-0.06 T$ and $t=0,1,2, \ldots,(T-1)$

$T=$ The number of bandpass filters ( $T=15$ is used in this study).

The energy concentrated in the $t-t h$ band is computed by

$E_{t}=\sum_{u=0}^{M-1} \sum_{v=0}^{N-1} B P_{E}(u, v) P(u, v)$

The normalized energy for the $t-t h$ bandpass filter is defined as

$P=\frac{E_{t}}{\sum_{t=0}^{T-1} E t}$

The energy concentration, $E_{C}$ is given by the entropy

$$
E_{C}=-\sum_{t=0}^{T-1} P \log P
$$

The maximum value of entropy is achieved when the distribution is uniform and it decreases when the distribution consists of a peak value. The QF is 


$$
\mathrm{QF}=\log T-E_{C}
$$

Good quality fingerprint leads to a higher value of QF and bad quality fingerprint leads to a lower value of QF [8]. 


\section{Appendix B- Experimental results of different enhancement algorithms using Quality Factor (QF) as an Evaluation Tool}

Table B-1

Values of QF for Different Fingerprint Image Enhancement Algorithms

\begin{tabular}{|c|c|c|c|c|}
\hline \multirow{2}{*}{$\begin{array}{l}\text { Fingerprint } \\
\text { Image } \\
\quad \#\end{array}$} & \multicolumn{4}{|c|}{ Quality Factor (QF) } \\
\hline & $\begin{array}{l}\text { Straight Gabor } \\
\text { Filter }\end{array}$ & $\begin{array}{l}\text { Curved Gabor } \\
\text { Filter }\end{array}$ & $\begin{array}{l}\text { Volterra } \\
\text { Filter }\end{array}$ & $\begin{array}{c}\text { Volterra Filter+ } \\
\text { Curved Gabor } \\
\text { Filter }\end{array}$ \\
\hline 1 & 0.7649 & 0.7740 & 0.7783 & 0.7814 \\
\hline 2 & 0.7781 & 0.7792 & 0.7820 & 0.7823 \\
\hline 3 & 0.7763 & 0.7770 & 0.7810 & 0.7821 \\
\hline 4 & 0.7753 & 0.7725 & 0.7808 & 0.7819 \\
\hline 5 & 0.7762 & 0.7740 & 0.7807 & 0.7811 \\
\hline 6 & 0.7762 & 0.7709 & 0.7816 & 0.7815 \\
\hline 7 & 0.7756 & 0.7769 & 0.7807 & 0.7817 \\
\hline 8 & 0.7755 & 0.7763 & 0.7802 & 0.7837 \\
\hline 9 & 0.7754 & 0.7801 & 0.7803 & 0.7821 \\
\hline 10 & 0.7759 & 0.7753 & 0.7802 & 0.7831 \\
\hline 11 & 0.7752 & 0.7786 & 0.7806 & 0.7817 \\
\hline 12 & 0.7748 & 0.7749 & 0.7810 & 0.7820 \\
\hline 13 & 0.7757 & 0.7779 & 0.7806 & 0.7810 \\
\hline 14 & 0.7760 & 0.7799 & 0.7807 & 0.7827 \\
\hline 15 & 0.7769 & 0.7782 & 0.7809 & 0.7843 \\
\hline 16 & 0.7752 & 0.7725 & 0.7807 & 0.7817 \\
\hline 17 & 0.7748 & 0.7801 & 0.7808 & 0.7808 \\
\hline 18 & 0.7747 & 0.7747 & 0.7808 & 0.7814 \\
\hline 19 & 0.7764 & 0.7770 & 0.7816 & 0.7817 \\
\hline 20 & 0.7764 & 0.7802 & 0.7812 & 0.7819 \\
\hline 21 & 0.7760 & 0.7730 & 0.7815 & 0.7820 \\
\hline 22 & 0.7770 & 0.7770 & 0.7814 & 0.7824 \\
\hline 23 & 0.7744 & 0.7793 & 0.7816 & 0.7815 \\
\hline 24 & 0.7742 & 0.7735 & 0.7817 & 0.7819 \\
\hline 25 & 0.7745 & 0.7738 & 0.7817 & 0.7827 \\
\hline 26 & 0.7746 & 0.7732 & 0.7813 & 0.7821 \\
\hline 27 & 0.7768 & 0.7805 & 0.7814 & 0.7805 \\
\hline 28 & 0.7762 & 0.7729 & 0.7816 & 0.7819 \\
\hline 29 & 0.7759 & 0.7768 & 0.7812 & 0.7820 \\
\hline 30 & 0.7743 & 0.7738 & 0.7816 & 0.7823 \\
\hline 31 & 0.7752 & 0.7783 & 0.7813 & 0.7813 \\
\hline 32 & 0.7761 & 0.7794 & 0.7810 & 0.7808 \\
\hline 33 & 0.7761 & 0.7799 & 0.7812 & 0.7815 \\
\hline 34 & 0.7749 & 0.7750 & 0.7814 & 0.7816 \\
\hline
\end{tabular}




\begin{tabular}{|l|l|l|l|l|}
\hline 35 & 0.7747 & 0.7805 & 0.7815 & 0.7815 \\
\hline 36 & 0.7754 & 0.7800 & 0.7817 & 0.7819 \\
\hline 37 & 0.7749 & 0.7714 & 0.7814 & 0.7823 \\
\hline 38 & 0.7755 & 0.7719 & 0.7815 & 0.7816 \\
\hline 39 & 0.7748 & 0.7763 & 0.7818 & 0.7817 \\
\hline 40 & 0.7758 & 0.7796 & 0.7816 & 0.7817 \\
\hline 41 & 0.7768 & 0.7808 & 0.7821 & 0.7824 \\
\hline 42 & 0.7757 & 0.7794 & 0.7816 & 0.7818 \\
\hline 43 & 0.7749 & 0.7790 & 0.7816 & 0.7812 \\
\hline 44 & 0.7751 & 0.7774 & 0.7811 & 0.7821 \\
\hline 45 & 0.7759 & 0.7781 & 0.7806 & 0.7811 \\
\hline 46 & 0.7763 & 0.7802 & 0.7808 & 0.7813 \\
\hline 47 & 0.7770 & 0.7784 & 0.7808 & 0.7813 \\
\hline 48 & 0.7749 & 0.7810 & 0.7815 & 0.7824 \\
\hline 49 & 0.7749 & 0.7785 & 0.7820 & 0.7826 \\
\hline 50 & 0.7754 & 0.7809 & 0.7815 & 0.7823 \\
\hline Mean & 0.7754 & 0.7770 & 0.7812 & 0.7819 \\
\hline Std & 0.0017 & 0.0013 & 0.0006 & 0.0005 \\
\hline
\end{tabular}




\section{References}

[1] Jain, L. Hong, S. Pankanti and R. Bolle, "An Identity-Authentication System using Fingerprint," Proceedings of the IEEE, vol. 85, no. 9, September 1997, pp 202-207.

[2] Newham, The Biometric Report. New York, NY: SJB Services, 1995.

[3] S. Thurnhofer and S.K. Mitra, "A General Framework for Quadratic Volterra Filters for Edge Enhancement," IEEE Transactions on Image Processing, vol. 5, no. 6, June 1996, pp 950-963.

[4] M. Kawagoe and A. Tojo, "Fingerprint Pattern Classification," Pattern Recognition, vol. 17, no. 3, pp. 295-303, 1984.

[5] L. Hong, Y. Wan and A. Jain, "Fingerprint Image Enhancement: Algorithm Analysis and Performance Evaluation," IEEE Transactions on Pattern Analysis and Machine Intelligence, vol. 20, no. 8, August 1998, pp 777-789.

[6] Jain, L. Hong, and R. Bolle, "On-Line Fingerprint Verification," IEEE Transactions on Pattern Analysis and Machine Intelligence, vol. 20, no. 8, August 1998, pp 302-314.

[7] K. Karu and A.K. Jain, "Fingerprint Classification," Pattern Recognition, vol. 29, no.3, pp. 389-404, 1996.

[8] D. Maltoni, D. Maio, A.K. Jain, S. Prabhakar, "Handbook of fingerprint recognition (Second Edition)," Springer, London, 2009.

[9] Maio and D. Maltoni, "Direct Gray-Scale Minutiae Detection in Fingerprints," IEEE Transactions on Pattern Analysis and Machine Intelligence, vol. 19, no. 1, January 1997, pp. 27-40.

[10] B.M. Mehtre, "Fingerprint Image Analysis for Automatic Identification," Machine Vision and Applications, 1993, pp. 124-139.

[11] L. O'Gorman and J.V. Nickerson, "An Approach to Fingerprint Filter Design," Pattern Recognition, vol. 22, no. 1, pp. 29-38, 1989.

[12] A. K. Jain, "Fundamentals of Digital Image Processing”, Englewood Cliffs, NJ: PrenticeHall, 1989.

[13] J. S. Lim, “2-D Signal and Image Processing”, Englewood Cliffs, NJ: Prentice-Hall, 1990.

[14] G. L. Sicuranza, "Quadratic filters for signal processing", Proc. IEEE, vol. 80, pp. 12631285, Aug. 1992.

[15] J. F. Kaiser, "Some Useful Properties of Teager's Energy Operator,” IEEE, 1993.

[16] D. Maltoni, D. Maio, A.K. Jain, S. Prabhakar, "Handbook of fingerprint recognition (Second Edition)," Springer, London, 2009.

[17] H.C. Lee and R.E. Gaensslen, Advances in Fingerprint Technology. New York, NY: Elsevier, 1991.

[18] V. K. Alilou, "Fingerprint matching: A simple approach." [Online]. Available: http://www.mathworks.com/matlabcentral/fileexchange/44369- fingerprint-matching-asimple-approach

[19] T. Kamei and M. Mizoguchi, "Image Filter Design for Fingerprint Enhancement," Proc. ISCV' 95, pp. 109-114, Coral Gables, Fla., 1995.

[20] D. Sherlock, D.M. Monro, and K. Millard, "Fingerprint Enhancement by Directional Fourier Filtering," IEE Proc. Visual Image Signal Processing, vol. 141, no. 2, pp. 87-94, 1994.

[21] A. Sherstinsky and R.W. Picard, "Restoration and Enhancement of Fingerprint Images Using M-Lattice: A Novel Non-Linear Dynamical System,” Proc. 12th ICPR-B, pp. 195-200, 1994.

[22] Moenssens, Fingerprint Techniques. London: Chilton Book Company, 1971. 
[23] X. Ming, W. Xiaopei, H. Quanping, "A Fast Thinning Algorithm for Fngerprint Image," ICISE, 2009.

[24] H. Tamura, "A Comparison of Online Thinning Algorithms from Digital Geometry Viewpoint," Proc of the $4^{\text {th }}$ Int. Conf. on Pattern Recognition, pp 715-719, 1978.

[25] P.E. Danielsson and Q. Z. Ye, "Rotation-Invariant Operators Applied to Enhancement of Fingerprints," Proc. Ninth ICPR, pp. 329- 333, Rome, 1988.

[26] K. Delac, M. Grgic, "A Survey of Biometric Recognition Method," 46th International SyrnPoSium Electronics in Marine, June 2004. 